\title{
Zrf1 is required to establish and maintain neural progenitor identity
}

\author{
Luigi Aloia, ${ }^{1}$ Bruno Di Stefano, ${ }^{1}$ Alessandro Sessa, ${ }^{2}$ Lluis Morey, ${ }^{1}$ Alexandra Santanach, ${ }^{1}$ \\ Arantxa Gutierrez, ${ }^{1}$ Luca Cozzuto, ${ }^{1}$ Salvador Aznar Benitah, ${ }^{1,3}$ Thomas Graf, ${ }^{1,3}$ Vania Broccoli, ${ }^{2}$ \\ and Luciano Di Croce ${ }^{1,3,4}$ \\ ${ }^{1}$ Centre for Genomic Regulation (CRG), Universitat Pompeu Fabra, 08003 Barcelona, Spain; ${ }^{2}$ Stem Cells and Neurogenesis Unit, \\ Division of Neuroscience, San Raffaele Scientific Institute, 20132 Milan, Italy; ${ }^{3}$ Institució Catalana de Recerca i Estudis Avançats \\ (ICREA), 08010 Barcelona, Spain
}

\begin{abstract}
The molecular mechanisms underlying specification from embryonic stem cells (ESCs) and maintenance of neural progenitor cells (NPCs) are largely unknown. Recently, we reported that the Zuotin-related factor 1 (Zrf1) is necessary for chromatin displacement of the Polycomb-repressive complex 1 (PRC1). We found that Zrf1 is required for NPC specification from ESCs and that it promotes the expression of NPC markers, including the key regulator Pax6. Moreover, Zrf1 is essential to establish and maintain Wnt ligand expression levels, which are necessary for NPC self-renewal. Reactivation of proper Wnt signaling in Zrf1-depleted NPCs restores Pax6 expression and the self-renewal capacity. ESC-derived NPCs in vitro resemble most of the characteristics of the self-renewing NPCs located in the developing embryonic cortex, which are termed radial glial cells (RGCs). Depletion of Zrf1 in vivo impairs the expression of key self-renewal regulators and Wnt ligand genes in RGCs. Thus, we demonstrate that Zrf1 plays an essential role in NPC generation and maintenance.
\end{abstract}

[Keywords: neural specification; Wnt ligands; embryonic stem cell]

Supplemental material is available for this article.

Received August 13, 2013; revised version accepted December 13, 2013.

Epigenetic regulators, such as proteins of the Polycombrepressive complex 1 (PRC1) and PRC2, repress developmental genes and play a crucial role in stem cells, in line with their essential role in embryonic development (Schuettengruber and Cavalli 2009; Aloia et al. 2013). We showed recently that the chromatin component Zuotin-related factor 1 (Zrf1) derepresses developmental genes by displacing the PRC1 complex from chromatin during differentiation of human teratocarcinoma cells (Richly et al. 2010). Furthermore, the Caenorhabditis elegans Zrf1 ortholog dnj11 was implicated in asymmetric cell division of neurosecretory motoneuron neuroblasts (Hatzold and Conradt 2008). Although these studies suggest that Zrf1 is possibly involved in embryonic development, whether it is involved in stem cell maintenance and differentiation has not yet been explored.

Mouse embryonic stem cells (mESCs) derived from embryonic day 3.5 (E3.5) blastocysts are a valid tool to study embryonic development since they are able to give rise to all cell types of the embryo in vivo and in vitro (Boiani and Scholer 2005; Niwa 2007; Nichols and Smith 2012). In the last few years, several experimental methods

${ }^{4}$ Corresponding author

E-mail luciano.dicroce@crg.eu

Article is online at http://www.genesdev.org/cgi/doi/10.1101/gad.228510.113. to efficiently generate neural progenitor cells (NPCs) from ESCs have been described (Conti et al. 2005; Colombo et al. 2006; Bibel et al. 2007). The first NPCs in the embryo are neuroepithelial cells present in the neural tube at E11.5. Neuroepithelial cells give rise to Pax6-positive radial glial cells (RGCs) that remain at the ventricular zone (VZ) of the embryonic cortex (Campbell and Gotz 2002; Malatesta et al. 2003, 2008; Gotz and Huttner 2005; Kriegstein and Alvarez-Buylla 2009). RGCs act as self-renewing cellular elements, undergoing symmetric proliferative divisions to maintain the pool of progenitors. RGCs also undergo asymmetric neurogenic division to generate more differentiated progenitors and to support migration of differentiating progenitors by acting as cellular scaffolding units. In adults, NPCs that originated from embryonic RGCs are present in restricted areas of the central nervous system (CNS), where they produce differentiated neurons and glia (Temple 2001; Merkle et al. 2007; Zhao et al. 2008; Miller and GauthierFisher 2009; Fuentealba et al. 2012). Importantly, NPCs

(C) 2014 Aloia et al. This article is distributed exclusively by Cold Spring Harbor Laboratory Press for the first six months after the fullissue publication date (see http://genesdev.cshlp.org/site/misc/terms. xhtml). After six months, it is available under a Creative Commons License (Attribution-NonCommercial 3.0 Unported), as described at http://creativecommons.org/licenses/by-nc/3.0/. 
are capable of repairing brain injuries upon transplantation (Temple 2001; Aboody et al. 2011).

Several transcription factors, including Sox $1 / 2$, Neurog $1 / 2$, and Brn $1 / 2$, are involved in neuroectodermal differentiation and specification of NPCs (Lee 1997; Aubert et al. 2003; Bani-Yaghoub et al. 2006; Suter et al. 2009; Gomez-Lopez et al. 2011). Moreover, the transcription factor Pax6 has been reported to be crucial for neural development and embryonic and adult NPC features, such as proliferation and differentiation (Stoykova et al. 1996; Gotz et al. 1998; Heins et al. 2002; Sansom et al. 2009; Tuoc et al. 2009). Several signaling pathways, such as Notch, Sonic hedgehog, and Wnt, are involved in the specification and maintenance of NPCs (Mizutani and Saito 2005; Han et al. 2008; Nusse 2008; Alvarez-Medina et al. 2009; Bluske et al. 2012; Bowman et al. 2013). During canonical Wnt signaling, $\beta$-catenin accumulates in the cytoplasm before translocating to the nucleus, where it can activate transcription (MacDonald et al. 2009). Wnt activity has been reported to antagonize the generation of NPCs from ESCs (Aubert et al. 2002; Haegele et al. 2003; Cajanek et al. 2009; Blauwkamp et al. $2012)$, although $\beta$-catenin was found to be required for neural differentiation (Otero et al. 2004; Lyashenko et al. 2011). Importantly, Wnt signaling is required for NPC maintenance (Chenn and Walsh 2002; Kalani et al. 2008) and differentiation into neurons (Hirabayashi et al. 2004; Lie et al. 2005). Recent evidence has shown that endogenously produced Wnt ligands are important for selfrenewal and multipotency of NPCs (Wexler et al. 2009). However, molecular mechanisms regulating Wnt ligand expression in NPCs are largely unknown.

Here we show that Zrf1 is essential for neuroectodermal specification and that it is required for NPC selfrenewal by regulating Pax6 expression. Moreover, we unveil a role for Zrf1 in the control of Wnt ligand expression in NPCs. We also demonstrate that in vivo Zrf1 depletion in the embryonic VZ of the cortex impairs the expression of key self-renewal regulators. We propose that Zrf1 is a key player required for first inducing NPC specification from ESCs and then maintaining NPC identity.

\section{Results}

Zrf1 is required for neuroectodermal specification of ESCs

To investigate whether Zrf1 has a role in ESCs, we stably depleted it from mESCs (Fig. 1A). We found that it did not affect either cell growth or the expression of pluripotency markers, such as Oct4, Nanog, Sox2, Rex1, and alkaline phosphatase (Fig. 1B; Supplemental Fig. S1A,B). To investigate the differentiation potential of Zrf1-depleted ESCs, we injected shZrf1 (Zrf1 shRNA) or shCtrl (control shRNA) cells into SCID mice to induce teratoma formation. Strikingly, immunostaining of teratomas derived from shZrf1 ESCs revealed a significant reduction of neuroectodermal markers, such as Nestin, $\beta 3$-tubulin, and GFAP (markers of NPCs, post-mitotic neurons, and astrocytes, respectively), whereas endodermal (FoxA2) and mesodermal (actin $\alpha$ smooth muscle [SMA]) markers were properly expressed (Fig. 1C; Supplemental Fig. S2A). In agreement with this, RT-qPCR analysis of teratomas indicated that Zrf1 depletion impaired the expression of neuroectodermal markers, including Nestin, Pax6, and Sox1, whereas mesodermal and endodermal markers were expressed at the same level or slightly higher in Zrf1-depleted teratomas (Fig. 1D). We thus decided to focus on the role of Zrf1 in neuroectodermal lineage specification.

To this end, we used an ESC line that expresses GFP under the control of the Sox1 promoter (Sox1-GFP ESCs), which is one of the earliest neuroectodermal markers induced in the embryo (Suter et al. 2009). Sox1-GFP ESCs were cultured in serum-free medium supplemented with $\mathrm{N} 2$ and B27 (N2B27 medium), which is known to promote neural induction (Ying et al. 2003). While control cells showed up-regulation of GFP expression, Zrf1 depletion significantly decreased the percentage of GFPpositive cells at days 3 and 5 in N2B27 medium (Fig. 1E). On the other hand, pluripotency markers were downregulated in shZrf1 cells cultured in N2B27 medium to the same level as in shCtrl cells (Supplemental Fig. S2B). Importantly, nonneural markers were not detectable in Zrf1-depleted cells grown in N2B27 medium at either the mRNA (data not shown) or protein (Supplemental Fig. S2C) level. In addition, Zrf1 depletion impaired the expression of the early neuroectodermal marker Nestin (Fig. 1F) and the marker of post-mitotic neurons, $\beta 3$ tubulin (Fig. 1G). To demonstrate that the absence of Zrf1 was responsible for the effects of the shRNAs, we introduced Flag-tagged human Zrf1 cDNA into Sox1-GFP ESCs depleted for Zrf1, thus restoring correct expression of Zrf1. As expected, re-expression of Zrf1 rescued the differentiation defect of shZrf1 ESCs (Fig. 1E-G).

To determine whether Zrf1 is also involved in other differentiation processes, we cultured shZrf1 cells in the presence of activin A for $5 \mathrm{~d}$ to induce the expression of mesodermal and endodermal markers. RT-qPCR analysis revealed that shZrf1 ESCs did not block the up-regulation of several mesodermal and endodermal markers in this culture condition (Supplemental Fig. S2D), suggesting that Zrf1 is specifically required for the ESCs to commit to neuroectodermal lineages.

\section{Zrf1 is essential for the generation of RGC-like NPCs from ESCs}

We next analyzed whether Zrf1 is involved in generating NPCs from ESCs by depleting Zrf1 from Sox1-GFP cells that were then treated with retinoic acid (RA) to induce their differentiation into Pax6-positive, RGC-like NPCs (Fig. 2A; Bibel et al. 2004, 2007). We found that depleting Zrf1 significantly down-regulated Sox1 (Supplemental Fig. S3A,B) but did not impair the expression of pluripotency markers as compared with control cells (Supplemental Fig. S3C). To elucidate the molecular function of Zrf1 during the generation of RGC-like NPCs, we performed gene expression profiling at day 6 of neural induction (see Supplemental Table 1). At this time point, Zrf1 depletion 
A

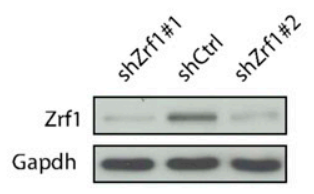

C

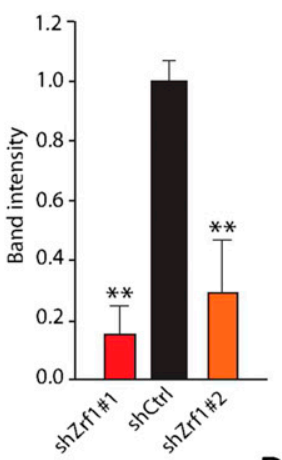

Immunostaining of teratomas

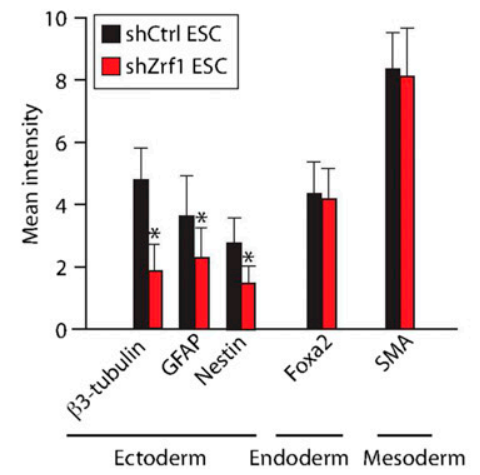

B

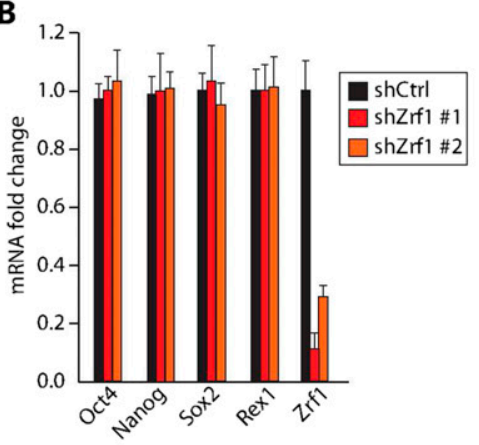

D

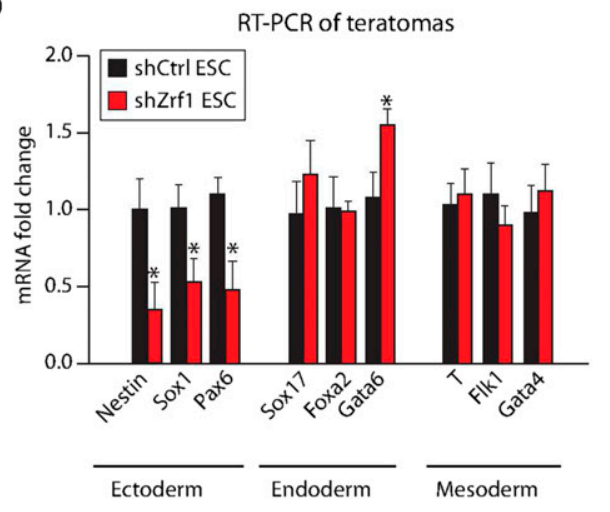

E Serum free medium supplemented with N2 and B27

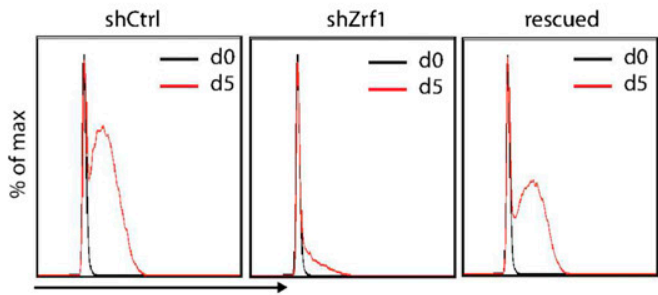

FITC (Sox1-GFP)

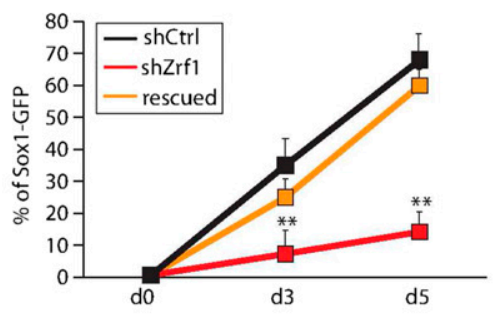

G

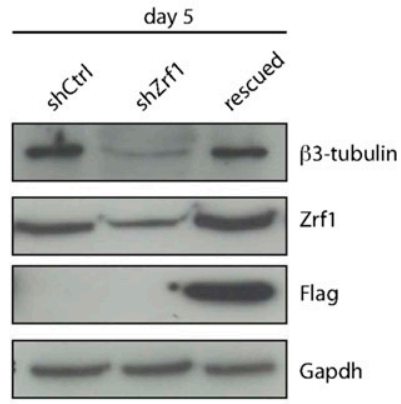

Figure 1. Zrf1 is required for neuroectodermal specification of ESCs. (A, left) Western blot for Zrf1 after infection of E14Tg2A ESCs with two different lentiviral shRNAs. (Right) Band intensity was quantified by using ImageJ software. Data represent the average of three independent experiments. $\left(^{\star \star}\right) P<0.01$. $(B)$ The relative mRNA expression of pluripotency markers after Zrf1 depletion. Values are normalized to Gapdh. Data represent the average of three independent experiments. $(C)$ Mean fluorescent intensity of immunostained cells in shCtrl or shZrf1 teratomas quantified by using ImageJ software is depicted in the graph. Data represent the average of five selected teratomas (10 fields for each teratoma). $\left(^{\star}\right) P<0.05$. $(D)$ The relative mRNA expression of selected markers of three germ layers in entire teratomas. Values are normalized to Gapdh. The analysis was performed on five different teratomas. $\left(^{\star}\right) P<0.05$. $(E$, left) FACS profile of Soxl-GFP expression ESCs grown in self-renewing condition (d0) and in N2B27 medium at day 5 (d5). (Right) The percentage of Sox1-GFP-positive cells at days 3 and 5 is reported in the graph. Data represent the average of three independent experiments. $\left.\left.\right|^{\star \star}\right) P<0.01 .(F)$ Nestin immunostaining at day 5 in N2B27 medium. Bar, $200 \mu \mathrm{m}$. The percentage of positive (as compared with total DAPI [40,60-diamidino-2-phenylindole]-positive) cells is reported in the picture. Data represent three independent experiments (seven fields were counted for each experiment). (G) Western blot for $\beta 3$-tubulin in shCtrl, shZrf1, and rescued cells (coexpressing shZrf1 and human Zrf1 cDNA tagged with 3xFlag) at day 5 in N2B27 medium. 
A

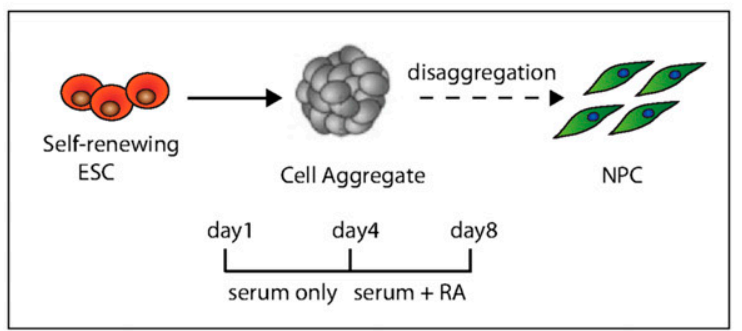

C

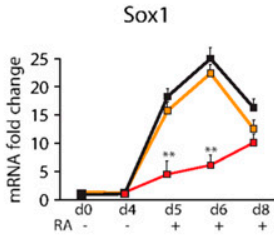

Nestin
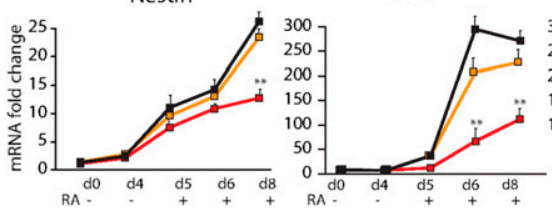

Neurod1

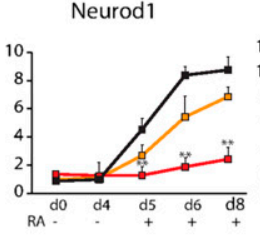

Brn2

Zrf1 occupancy
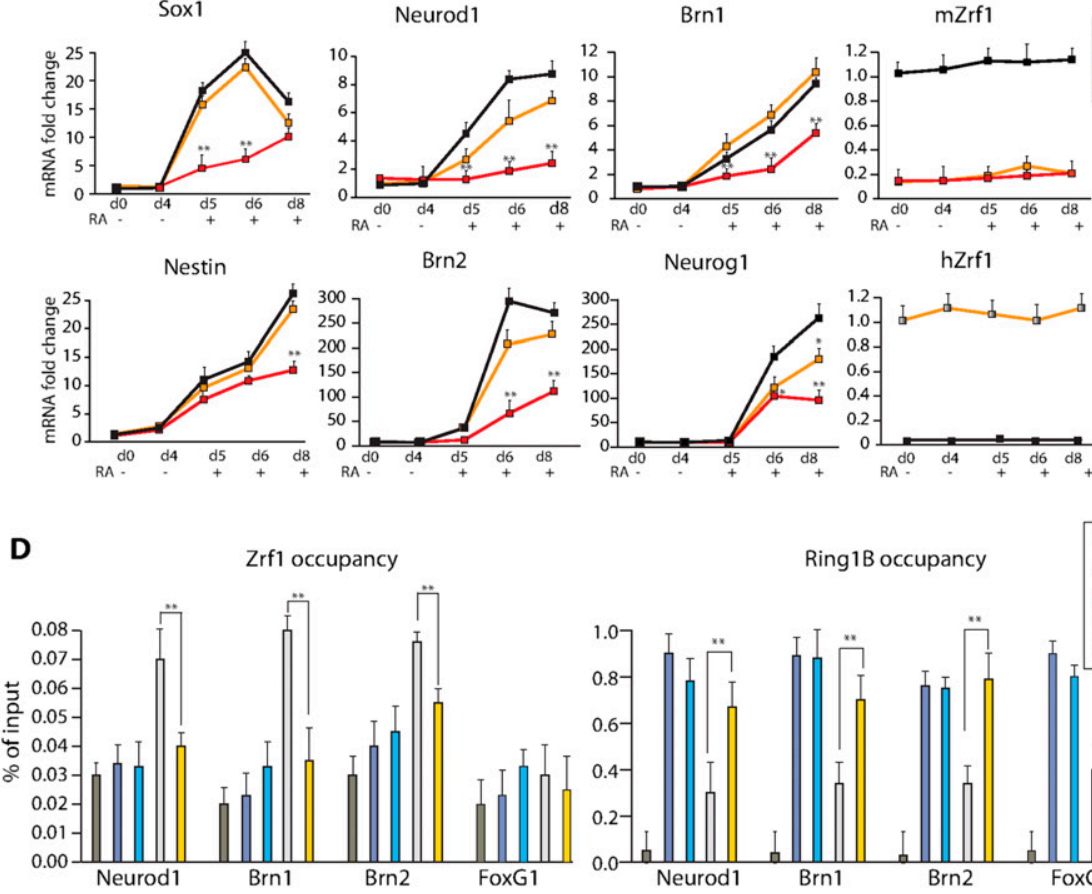

Neurog1

B

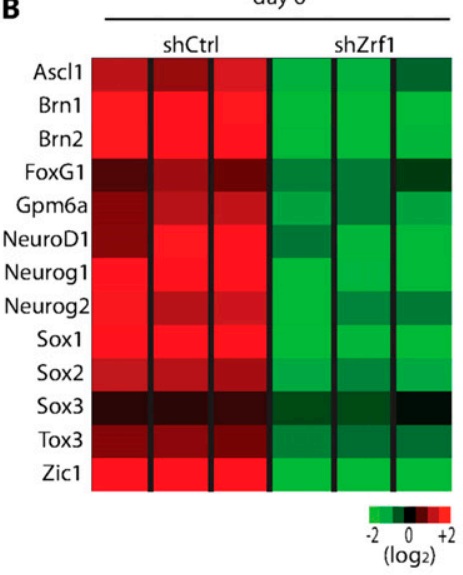

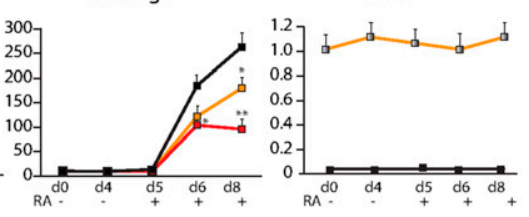

E
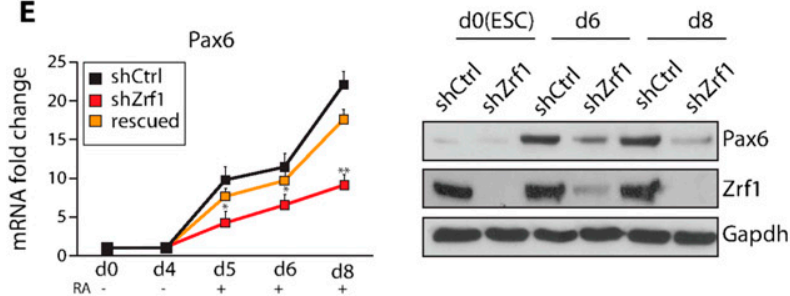

Figure 2. Zrf1 is required for generation of RGC-like NPCs from ESCs. (A) Scheme of generation of RGC-like NPCs. Briefly, ESCs were grown as cell aggregates (CAs) in medium supplemented with $10 \%$ serum. At day 4, RA was added to the medium to promote the generation of Pax6-positive RGC-like NPCs. At day 8, CAs were disaggregated, and RGC-like NPCs were harvested for the analysis. $(B)$ Heat map illustrating the expression changes of selected NPC markers in shCtrl and shZrf1 CAs at day 6 of neural induction. Each lane corresponds to an independent biological sample. $(C)$ The relative mRNA expression of NPC markers during generation of RGC-like NPCs. Values are normalized to Gapdh. Data represent the average of three independent experiments. $\left({ }^{\star}\right) P<0.05 ;\left(^{\star \star}\right) P<0.01 .(D) \mathrm{Zrf} 1$ ChIP-qPCR (left panel) and Ring1B ChIP-qPCR (right panel) of selected NPC markers in ESCs (d0) and CAs at day 6 of neural specification (d6). Values are expressed as percentage of input. Data represent three different experiments. $\left(^{\star \star}\right) P<0.01 .(E)$ Pax6 expression at mRNA (left panel) and protein (right panel) levels during NPC specification from ESCs. The graph represents data from three different experiments. $\left.\left(^{\star}\right) P<0.05 ;\left.\right|^{\star \star}\right) P<0.01$. 
up-regulated 1315 genes and down-regulated 1118 genes (Supplemental Fig. S3D). Gene ontology (GO) analysis revealed that the down-regulated genes were enriched for neuroectoderm markers and were mainly involved in transcription and embryonic development. On the other hand, a significant enrichment of markers of nonneural lineages was observed in the up-regulated gene set (Supplemental Fig. S3D). Specifically, Zrf1 knockdown impaired the expression of several NPC key regulators, including Sox1, Neurog1, Neurog2, and Neurod1 (Fig. 2B). Moreover, Zrf1 depletion affected the expression of markers of the forebrain (FoxG1), midbrain (including Lmx1A and Pitx3), and hindbrain (including HoxB4 and HoxB8) (Supplemental Fig. S3E). As expected, Zrf1 reexpression restored the expression of the mentioned genes (Fig. 2C).

Chromatin-bound Zrf1 increases with RA treatment (Richly et al. 2010), leading us to hypothesize that Zrf1 is recruited to NPC genes upon ESC differentiation. Indeed, chromatin immunoprecipitation (ChIP) analysis revealed that Zrf1 was recruited to the promoter regions of several NPC markers, such as Neurod1, Brn1, and Brn2 (Fig. 2D, left panel). To investigate whether Zrf1 is involved in derepressing neural genes, we studied the occupancy of the PRC1 subunit Ring1B at Zrf1-bound genes. ChIP experiments followed by RT-qPCR indicated that Ring1B was bound to Zrf1 target genes in ESCs, whereas Ring1B occupancy decreased in differentiating cells (Fig. 2D, right panel). However, in Zrf1-depleted ESCs, Ring1B occupancy at Zrf1 targets was maintained also in differentiating cells (Fig. 2D, right panel). Taken together, these data suggest that Zrf1 is essential for PRCl displacement on selected NPC markers and their transcriptional activation upon differentiation.

Finally, we found that Pax6 expression in shZrf1 cells was slightly reduced at day 6 and further down-regulated at day 8 of NPC specification at both the mRNA and protein levels as compared with shCtrl cells (Fig. 2E). This indicated that Zrf1 is necessary to generate Pax6-positive NPCs from ESCs.

\section{Zrf1 regulates Pax6 expression and is essential for self-renewal of NPCs}

Since Zrf1 was crucial for the up-regulation of several NPC markers during NPC specification and thus for their expression in NPCs, we hypothesized that it could also have a role in the maintenance of NPC features. To test this hypothesis, we generated self-renewing NPCs from wild-type ESCs that could be maintained for $>50$ passages in culture (Colombo et al. 2006). These self-renewing NPCs were then infected with Ctrl or Zrf1 shRNAs. Zrf1depleted NPCs grew slower than shCtrl NPCs (Fig. 3A), and Zrf1 depletion significantly decreased cell proliferation as measured by BrdU incorporation (Fig. 3B). On the other hand, no changes in the percentage of apoptotic cells were observed after Zrf1 knockdown (Supplemental Fig. S4A). At the molecular level, Zrf1 depletion resulted in the down-regulation of several genes involved in maintaining NPC identify, such as Pax6, Emx2, Hes5,
Nkx2.1, and Dlx1 (Fig. 3C), but did not affect other NPC markers, such as Sox2 and SSEA1 (Fig. 3C; Supplemental Fig. S4B). The specificity of the shRNAs in NPCs was verified by using a vector encoding the human Zrf1 cDNA. Re-expression of Zrf1 restored the correct levels of NPC markers in shZrf1 NPCs (Supplemental Fig. S4C).

The self-renewal capability of NPCs is characterized by sphere formation. Strikingly, Zrf1 depletion significantly affected primary and secondary sphere formation (Fig. 3D; Supplemental S4D). Importantly, re-expression of correct Zrf1 levels restored the expression of NPC markers and overcame the self-renewal defect (Supplemental Fig. S4D), indicating that Zrf1 is essential for selfrenewal of NPCs.

Since Pax6 is the key self-renewal regulator of embryonic and adult NPCs (Sansom et al. 2009), we checked whether re-expression of Pax6 in shZrf1 NPCs was able to restore proper self-renewal capacity. We expressed a Flagtagged mouse Pax6 cDNA in shCtrl and shZrf1 NPCs. Pax6 levels in shCtrl NPCs were slightly over-expressed with respect to the normal endogenous levels. Importantly, overexpression of Pax6 in shZrf1 NPCs restored its levels similar to that observed endogenously (Fig. 3E). Pax6-shZrf1 NPCs properly formed primary and secondary spheres, thus exhibiting correct self-renewal features (Fig. 3F,G). Thus, we conclude that Pax6 down-regulation was responsible for the self-renewal defect in Zrf1depleted cells. Since Pax6 has been reported to be crucial for not only self-renewal but also neuronal differentiation (Gotz et al. 1998; Lie et al. 2005), we checked whether Zrf1-depleted NPCs could still differentiate toward postmitotic neurons. We found that Zrf1 depletion impaired the generation of $\beta 3$-tubulin-positive neurons when NPCs were grown either in the absence of EGF and FGF2 (Supplemental Fig. S4E) or with a B27 supplement (Supplemental Fig. S4F), indicating that Zrf1 also plays a role in NPC differentiation. Pax6 re-expression significantly improved the differentiation capacity of shZrf1 NPCs (Supplemental Fig. S4F). Taken together, these results indicate that Zrf1 is required for both self-renewal and differentiation in NPCs.

\section{A PRC1-dependent Zrf1 involvement in neurogenesis}

To gain insight into Zrf1 function, we next performed ChIP experiments followed by massive parallel sequencing (ChIP-seq) of Zrf1 in the two stem cell populations of ESCs and NPCs. We did not find significant Zrf1-binding sites in ESCs $(\sim 150$ peaks, false discovery rate $[\mathrm{FDR}]=$ $100 \%$ ), whereas we found $\sim 12,000$ significant peaks (FDR $<5 \%$ ) corresponding to 5989 genes in NPCs (see Supplemental Table 2). GO analysis of Zrf1 target genes showed that they were significantly enriched for neural lineages (Fig. 4A). To elucidate a possible PRC1-dependent role of Zrf1 in neurogenesis at a genome-wide level, we took advantage of previously published Ring1B ChIP-seq in ESCs (Morey et al. 2012) and performed Ring1B ChIPseq in NPCs. Ring1B occupied 5535 genes in ESCs and $>10,000$ genes in NPCs (see Supplemental Table 2). As expected, Ring1B target genes were enriched for different 
A

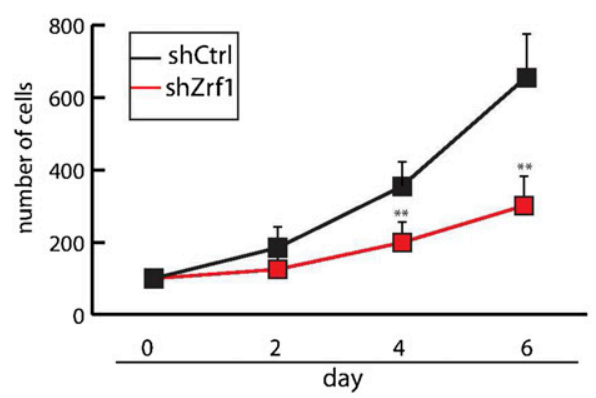

C

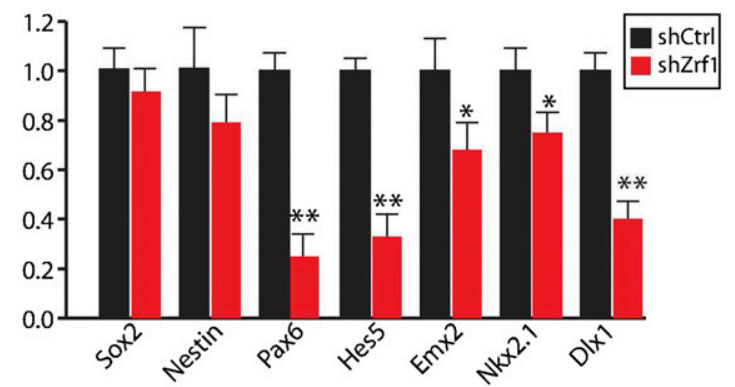

E
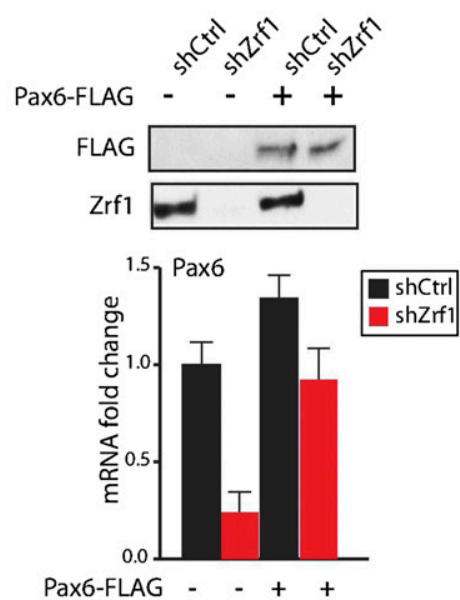

$\mathbf{F}$

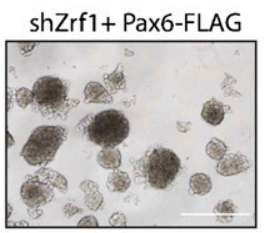

B
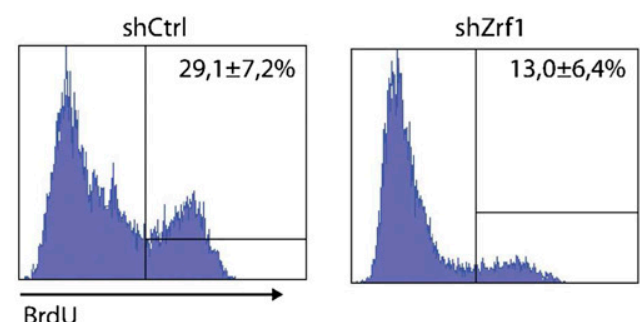

D

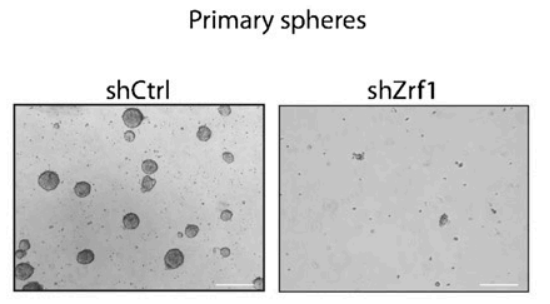

G
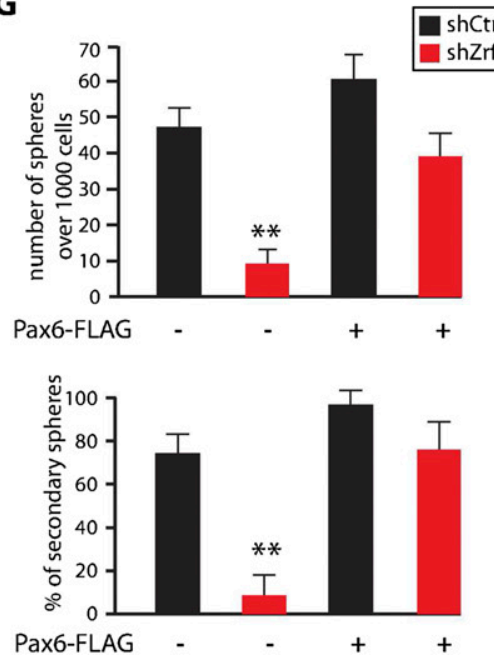

Figure 3. Zrf1 is required for self-renewal of ESC-derived NPCs. $(A)$ Growth curve of shCtrl and shZrf1 NPCs and rescued NPCs. Data are representative of three independent experiments. $\left(^{\star}\right) P<0.05 ;\left(^{\star \star}\right) P<0.01$. (B) FACS plot of BrDU staining after incubation of 20 min in shCtrl and shZrf1 NPCs. Data are representative of three independent experiments. $(C)$ The relative mRNA expression of several NPC markers in shCtrl and shZrf1 NPCs. Values are normalized to Gapdh. Data represent the average of three independent experiments. $\left(^{\star}\right) P<0.05 ;\left(^{\star \star}\right) P<0.01$. (D) Representative pictures of shCtrl and shZrf1 NPCs grown as spheres. Bars, $400 \mu \mathrm{m}$. $(E, t o p$ panel) Western blot of Flag and Zrf1 expression in shCtrl and shZrf1 NPCs electroporated with Flag-tagged Pax6 cDNA. (Bottom panel) The relative mRNA expression levels of Pax6 in shCtrl and shZrf1 after Pax6 overexpression. $(F)$ Representative pictures of shCtrl and shZrf1 NPCs after Pax6 overexpression grown as spheres. Bars, $250 \mu \mathrm{m}$. (G, top panel) Number of shCtrl and shZrf1 NPCs overexpressing Pax6 that gave rise to spheres over 1000 cells. (Bottom panel) Percentage of cells derived by disaggregation of primary spheres able to give rise to secondary spheres over the total number of cells. Data are representative of five different experiments. $\left({ }^{\star \star}\right) P<0.01$.

lineages, including mesodermal, neural, and embryonic lineages (Fig. 4A,B). Since most of the Ring1B-binding sites were found at the transcriptional start site (TSS), we analyzed only peaks at TSSs for both Ring1B and Zrf1.
Comparison of Zrf1-binding sites in NPCs and Ring1B in ESCs indicated that common targets were mostly enriched for neural lineages (Fig. 4A), suggesting that Zrf1 is mainly recruited to PRCl targets related to neurogenesis during NPC specification. Moreover, a significant enrichment 
Aloia et al.

A

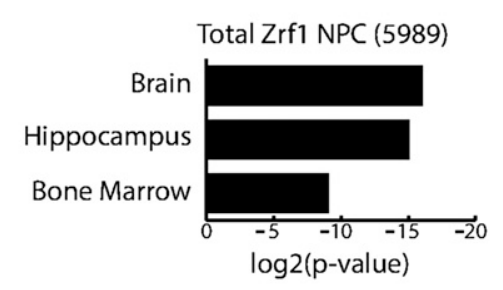

Only Zrf1 (1380)
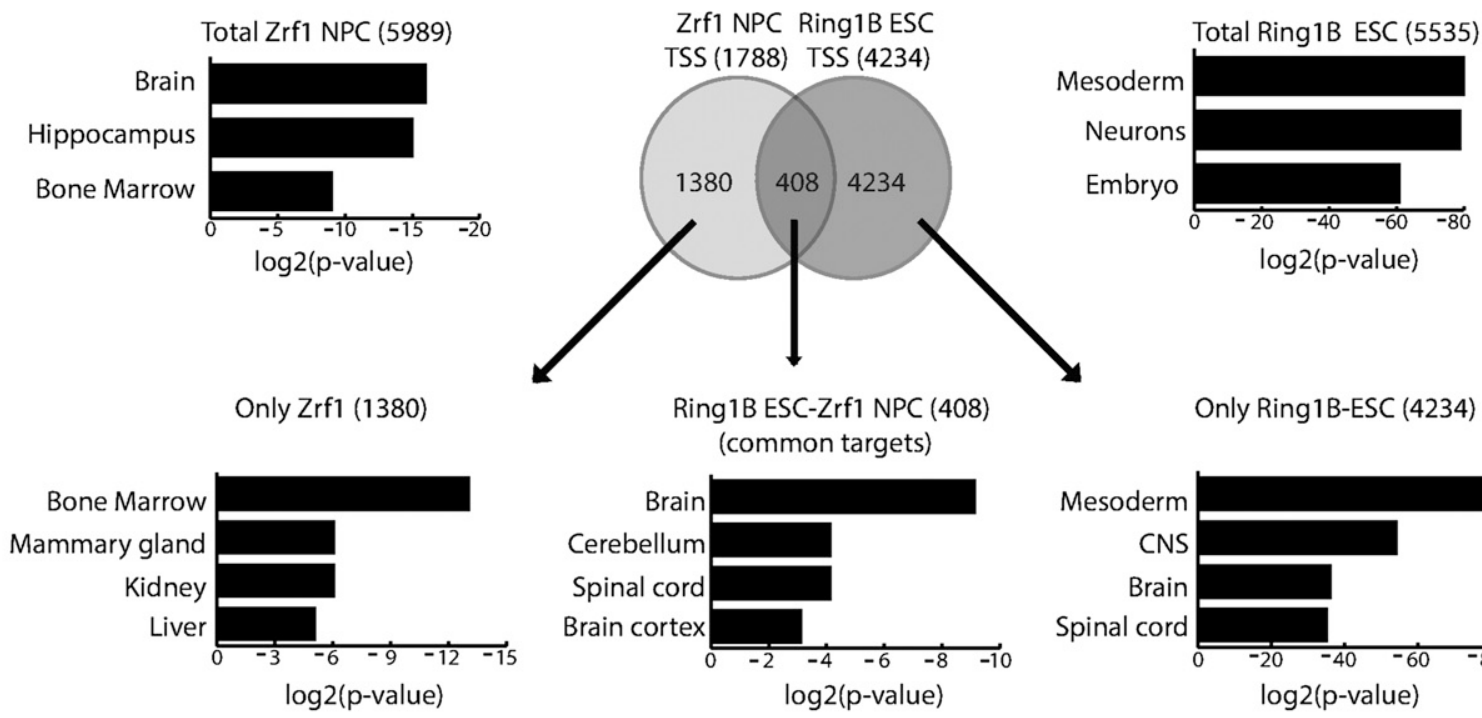

B

C

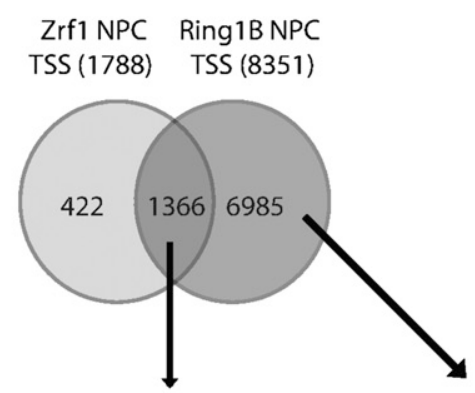

Total Ring1B NPC (10202)
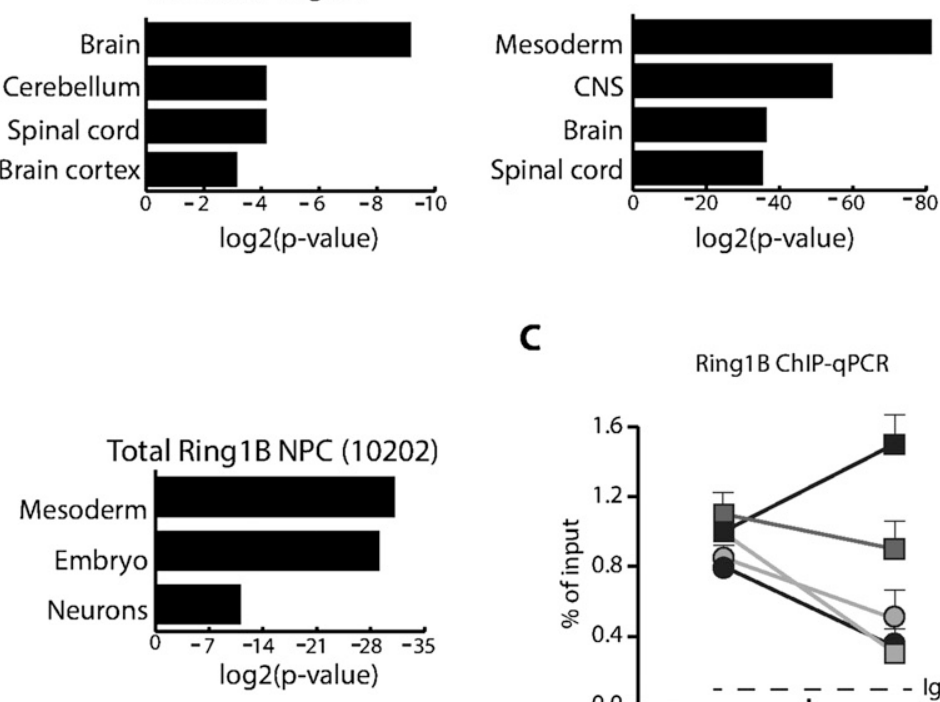

Only Ring1B-ESC (4234)

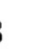

Ring1B ChIP-qPCR

Ring1B NPC-Zrf1 NPC (1366)

Only Ring1B-NPC (6985)
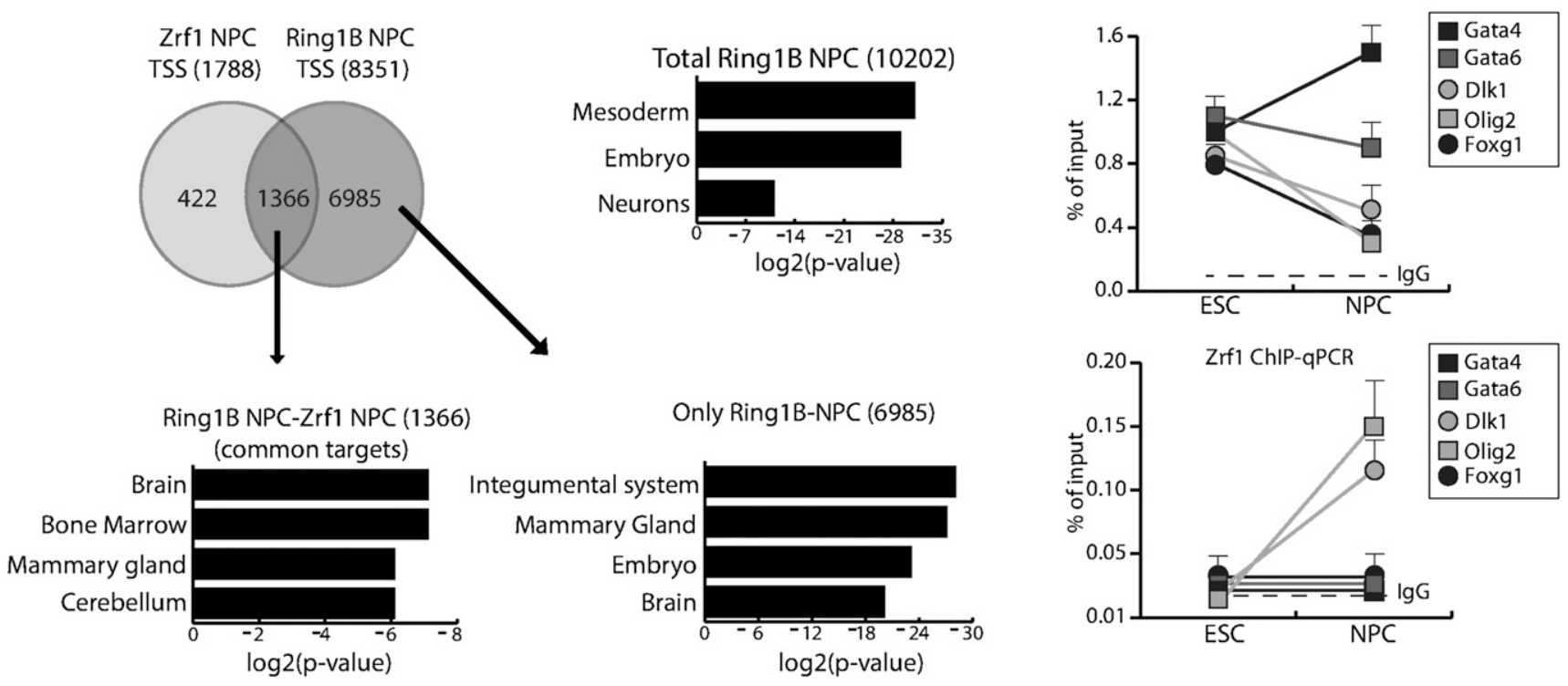

Figure 4. A PRC1-dependent role of Zrf1 is involved in neurogenesis. $(A)$ Overlap between Ring1B targets in ESCs and Zrf1 targets in NPCs. GO analysis reports enrichment in expression of total Ring1B and Zrf1 targets, common targets, and targets occupied by only Ring1B or Zrf1. GO analysis was performed by using Genomatix or DAVID software. $(B)$ Overlap between Ring1B and Zrf1 targets in NPCs. GO analysis reports enrichment in expression of total Ring1B targets, common targets, and targets occupied by only Ring1B or Zrf1. GO analysis was performed by using Genomatix or DAVID software. (C) Ring1B ChIP-qPCR (top panel) and Zrf1 ChIP-qPCR (bottom panel) of selected genes in ESCs and NPCs. Values are reported as percentage of input. Data represent three different experiments. $\left(^{\star \star}\right) P<0.01$.

for neural lineages was also observed in PRC1 targets not bound by Zrf1 (Fig. 4A), indicating that PRC1 also exerts a Zrf1-independent function in neurogenesis. By comparing common target genes of Zrf1 and Ring1B in NPCs, we found that most of the Zrf1 targets colocalize with Ring1B and were significantly enriched for neural lineages (Fig. 4B). In line with this, we found that Ring1B occupancy was lower in NPCs as compared with ESCs on proneural genes (such as Dlk1 and Olig2) that were bound by Zrf1 in NPCs (Fig. 4C; Supplemental Fig. S5A). Notably, Ring1B occupancy decreased also on the proneural gene FoxG1, which was not bound by Zrf1 (as also shown in Fig. 2D), confirming a Zrf1-independent role for PRC1 in neurogenesis (Fig. 4C). On the other hand, Ring1B occupancy 
was not affected on nonneural genes (such as Gata4 and Gata6) that were not bound by Zrf1 (Fig. 4C). Finally, we observed that a subset of Zrf1 targets was not bound by PRC1 in either ESCs or NPCs and that these were enriched for nonneural lineages (Supplemental Fig. S5B), suggesting a possible PRC1-independent function of Zrf1 in nonneural lineages. Taken together, these data highlight a PRC1-dependent role of Zrf1 in neurogenesis.

\section{Zrf1 controls the expression of selected Wnt ligands in NPCs}

Among the Zrf1 targets in NPCs, we found several genes involved in different signaling pathways, such as Pdgfra, Dlk1, Dclk1, and several Wnt ligands. ChIP-qPCR analysis confirmed that Zrf1 was bound to these genes in NPCs (Supplemental Fig. S5C-E). Since the autocrine/ paracrine Wnt signaling generated by endogenous Wnt ligands plays a crucial role in controlling NPC selfrenewal (Kalani et al. 2008; Wexler et al. 2009), we hypothesized that Zrf1 could regulate Wnt ligand genes. We thus analyzed the expression levels of Zrf1-bound Wnt ligands in ESCs, NPCs, and neurons. We found that several Zrf1-bound Wnt ligands, such as Wnt1, Wnt3, $W n t 5 b$, and Wnt6, were significantly up-regulated in NPCs as compared with ESCs. In addition, Wnt3 and Wnt6 were further up-regulated in neurons as compared with NPCs, whereas $W n t 7 b$ was specifically up-regulated in neurons (Fig. 5A). Since Wnt signaling impairs NPC specification from ESCs (Aubert et al. 2002; Haegele et al. 2003; Cajanek et al. 2009; Blauwkamp et al. 2012), we analyzed the expression of active $\beta$-catenin at different developmental stages (e.g., in ESCs, during NPC specification, in self-renewing NPCs, and in post-mitotic neurons). In line with previous reports, the amount of active $\beta$-catenin decreased during NPC specification (Supplemental Fig. S5F), indicating that the up-regulation of selected Wnt ligands in NPCs as compared with ESCs is not functional during NPC specification. To investigate whether Zrf1 was directly involved in inducing the expression of the mentioned Wnt ligands, we performed Zrf1 and Ring1B ChIP in shCtrl and shZrf1 NPCs. Interestingly, Zrf1 binding on all of the Wnt ligands analyzed significantly decreased in post-mitotic neurons as compared with NPCs, suggesting that Zrf1 regulates Wnt ligand genes specifically in NPCs (Fig. 5B). As expected, we found that Ring1B occupancy in control cells was significantly lower in NPCs and neurons as compared with ESCs. On the other hand, Ring1B occupancy remained significantly higher in shZrf1 NPCs (Fig. 5C) as compared with control NPCs, suggesting that Zrf1 plays an important role for Ring1B displacement upon differentiation of ESCs into NPCs. Moreover, we found that several Zrf1-bound Wnt ligands were significantly down-regulated in Zrf1-depleted NPCs as compared with control cells at the mRNA and protein levels (Fig. 5D,E). Taken together, these data indicate that Zrf1 is necessary to establish and maintain correct expression levels of selected Wnt ligands in NPCs.
Regulation of Wnt signaling mediated by Zrf1 controls NPC self-renewal

To assess whether the down-regulation of Wnt ligands observed in Zrf1-depleted NPCs impaired the canonical Wnt signaling, we analyzed the amount of active $\beta$-catenin in shZrf1 NPCs. Zrf1 depletion resulted in decreased amounts of active $\beta$-catenin, indicating that canonical Wnt signaling was impaired in shZrf1 NPCs (Fig. 6A). We therefore hypothesized that the impairment of the Wnt signaling observed in shZrf1 NPCs could account for the self-renewal defects observed after Zrf1 depletion. To test this hypothesis, shZrf1 NPCs were treated with the GSK3 inhibitor CHIR99021 (CHIR), which is able to activate $\beta$-catenin in the absence of Wnt ligand stimulation (Fig. 6A; Ying et al. 2008). Since our results indicated that down-regulation of Pax6 is responsible for the selfrenewal defect of Zrf1-depleted NPCs (Fig. 3), we investigated the level of Pax6 expression after CHIR treatment. Importantly, CHIR treatment at least partially rescued the expression level of Pax6 in shZrf1 NPCs at both mRNA and protein levels (Fig. 6A,B), confirming that Pax6 is a downstream target of Zrf1 in NPCs. In line with this, CHIR treatment resulted in a significant increase in the number of spheres generated by shZrf1 NPCs (Fig. 6C). However, these spheres were smaller than control spheres, a finding compatible with the incomplete rescue of Pax6 expression. CHIR treatment was able to completely rescue the capability of shZrf1 NPCs to give rise to secondary spheres, thus restoring their selfrenewal capacity (Fig. 6D). Partial rescue of Pax6 expression and correct self-renewal features were also observed in shZrf1 NPCs cultured with recombinant Wnt3a (Fig. 6B-D) or R-spondin (data not shown). On the other hand, CHIR treatment was not able to restore the differentiation defect of shZrf1 NPCs (Supplemental Fig. S5G). Taken together, these results unveil a novel role for Zrfl in Wnt signaling and indicate that it is essential for NPC self-renewal.

Since Wnt signaling controls proliferation and differentiation of several stem cell types, we investigated a possible role for Zrf1 in nonneural stem cell lineages. We performed Zrf1 knockdown in primary mesenchymal stem cells (MSCs) (Supplemental Fig. S6A-C), the myoblast cell line C2C12 (Supplemental Fig. S6D-F), and the ESC-derived hematopoietic progenitor cell line HPC7 (Supplemental Fig. S7); these stem cell lineages are dependent on Wnt signaling. We observed that Zrf1 depletion slightly decreased the growth rate of these stem cells, yet differentiation capability was not affected, suggesting that Zrf1 exerts a specific role in NPCs.

\section{Zrf1 regulates the expression of Wnt ligands and NPC master genes in vivo}

To investigate Zrf1 function in NPCs in vivo, we introduced either the shCtrl or shZrf1 vector with a GFPexpressing vector to cells in the VZ of an E13.5 cortex (Fig. 7A). At this developmental stage, the VZ contains Pax6-positive NPCs with RGC features. We then analyzed the GFP-positive regions from E15.5 embryos (e.g., 
Aloia et al.

A

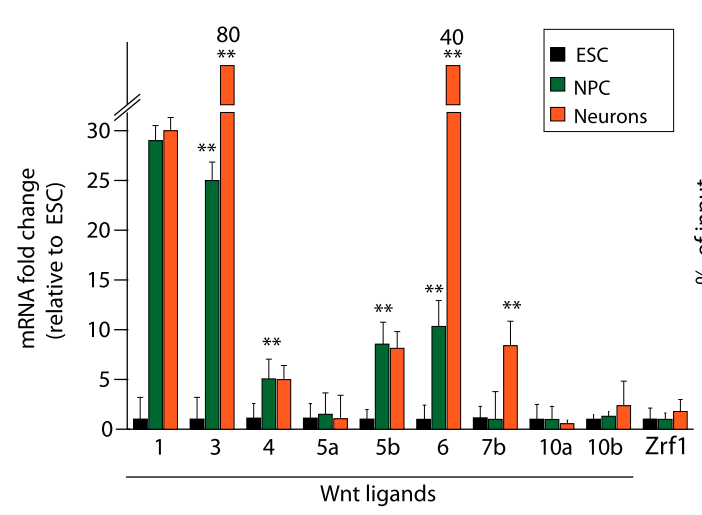

C

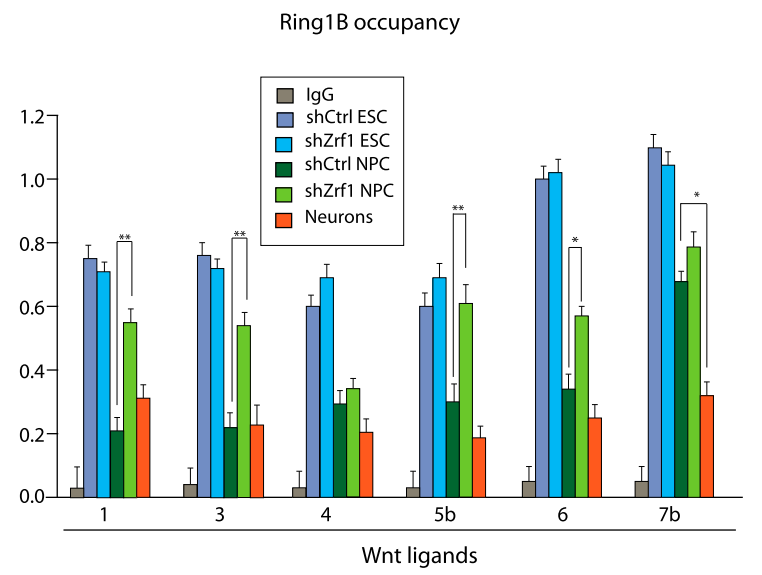

B

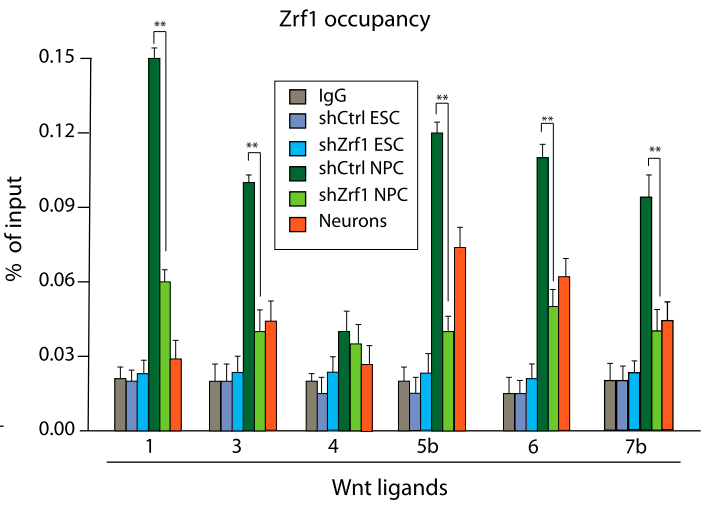

D

$\mathbf{E}$
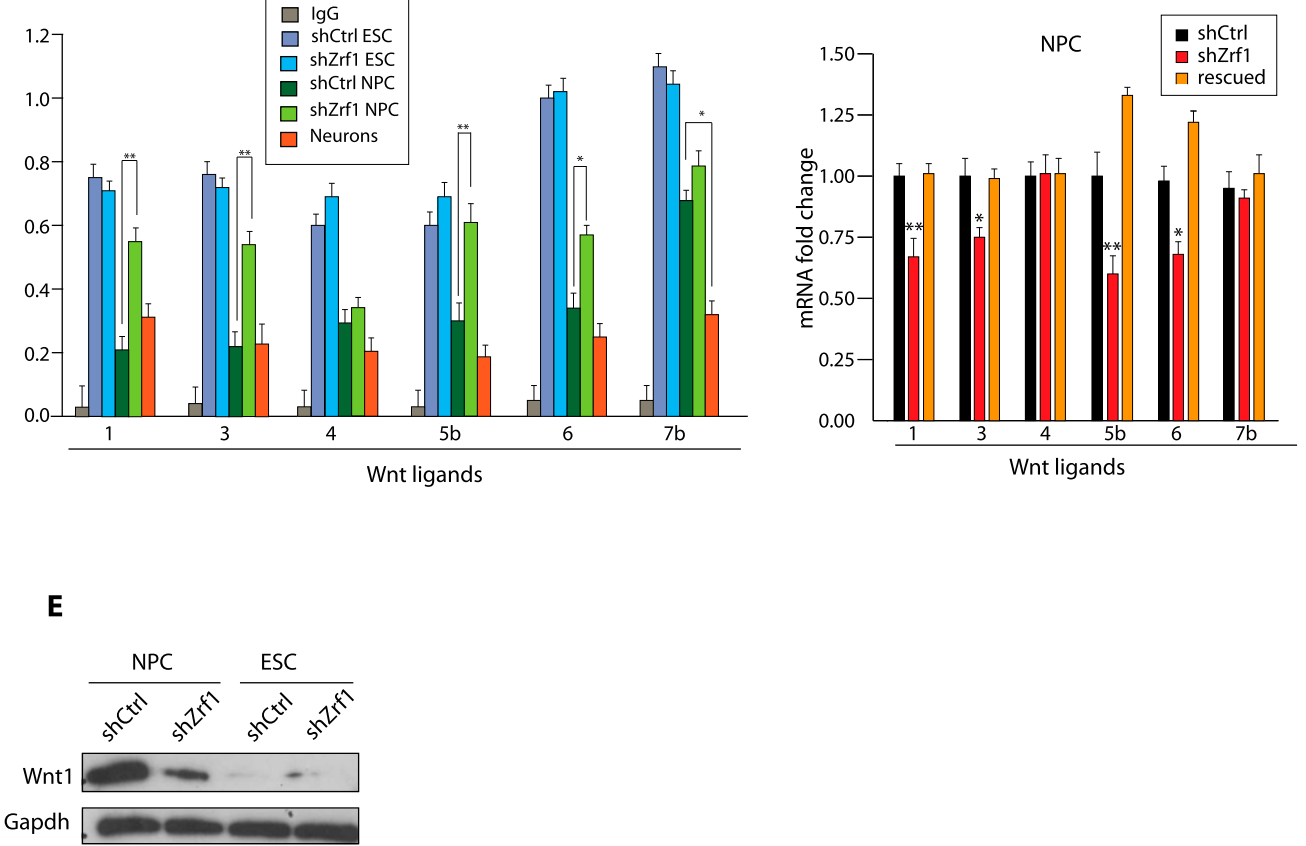

Figure 5. Zrf1 controls the expression of several Wnt ligands in NPCs. (A) The relative mRNA expression of Zrf1-bound Wnt ligands in ESCs, NPCs, and post-mitotic neurons. Data are representative of three different experiments. $\left(^{\star \star}\right) P<0.01$. $(B) \mathrm{Zrf1}$ ChIP-qPCR of selected Wnt ligands in ESCs, NPCs, and post-mitotic neurons. Values are expressed as percentage of input. Data represent three different experiments. $\left(^{\star \star}\right) P<0.01$. $(C)$ Ring1B ChIP-qPCR of selected Wnt ligands in ESCs, NPCs, and post-mitotic neurons. Wnt4 was used as control. Values are expressed as percentage of input. Data represent three different experiments. $\left(^{\star \star}\right) P<0.01$. $(D)$ The relative mRNA expression level of Zrf1-bound Wnt ligands in NPCs as compared with ESCs. $\left(^{\star \star}\right) P<0.01$. $(E)$ Western blot of Wnt1 expression in shCtrl and shZrfl ESCs and shCtrl and shZrf1 NPCs.

$48 \mathrm{~h}$ later). Interestingly, the number of shZrf1 cells was significantly reduced as compared with shCtrl cells (Fig. 7B; Supplemental Fig. S8A). To test whether the reduction of shZrf1 cells could be due to a proliferation defect, we stained the cells with a Ki67 antibody, which marks actively proliferating cells. We found that Zrf1 knockdown cells had fewer Ki67-positive cells (Supplemental Fig. S8B). On the other hand, we did not observe differences in cell death, as a TUNEL assay showed no changes in shCtrl and shZrf1 embryonic cortices between GFP/ shCtrl and GFP/shZrf1 cells (Supplemental Fig. S8C).
Finally, we investigated the migration capability of electroporated cells as an indication of the differentiation process. The number of GFP-positive cells able to migrate toward the proper cortical plate was severely reduced after Zrf1 depletion (Fig. 7C), suggesting that Zrf1 is necessary in RGC progeny to sustain a correct pattern of radial migration during cerebral cortex development.

To gain insight into the Zrf1 molecular function in the embryonic cortex, we sorted GFP-positive cells $48 \mathrm{~h}$ after electroporation with shCtrl- and shZrf1-encoding vectors. By analyzing these cell fractions by RT-qPCR, we 

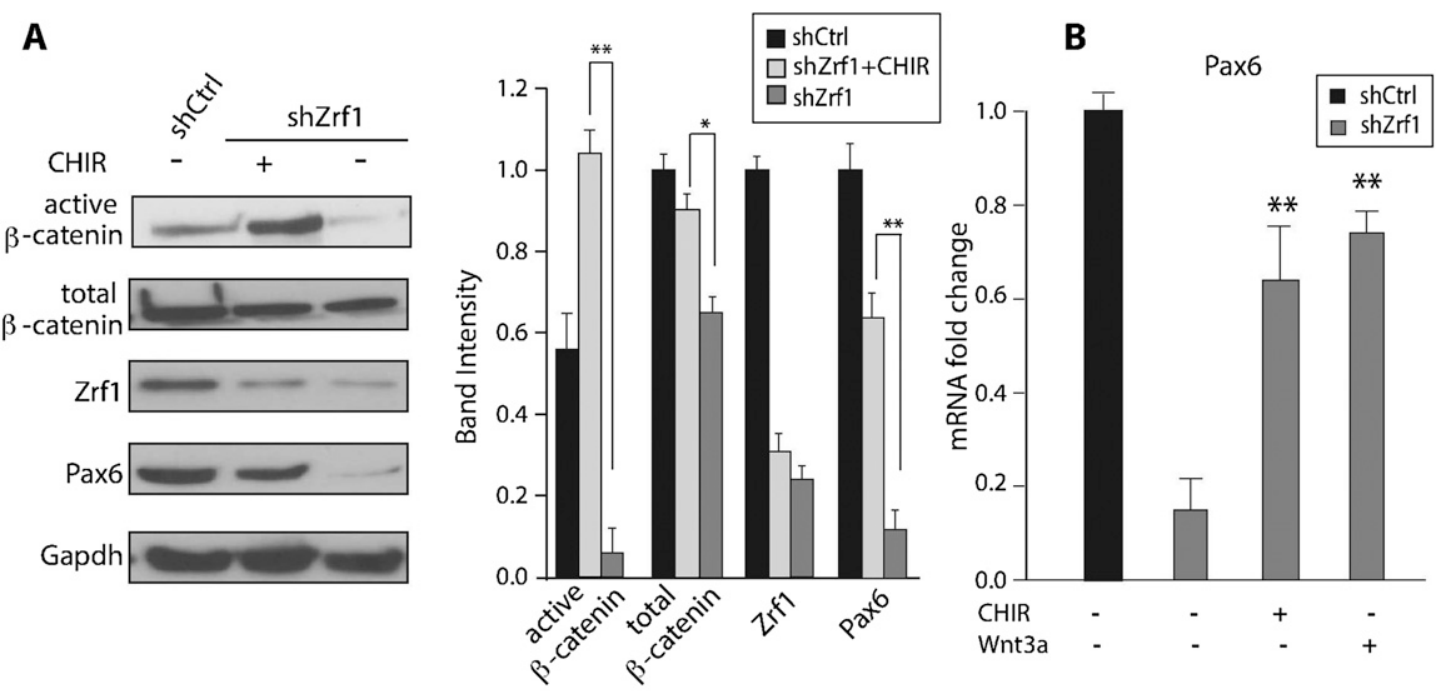

C

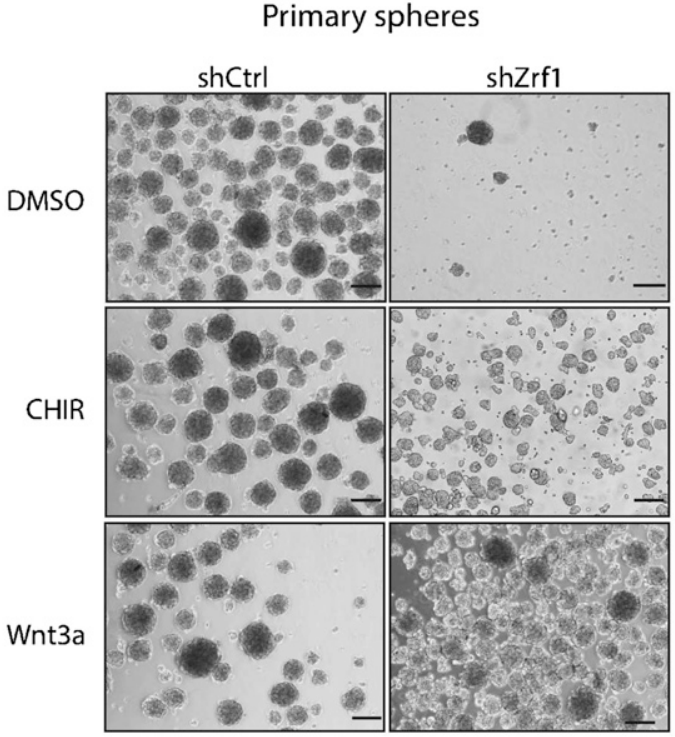

D
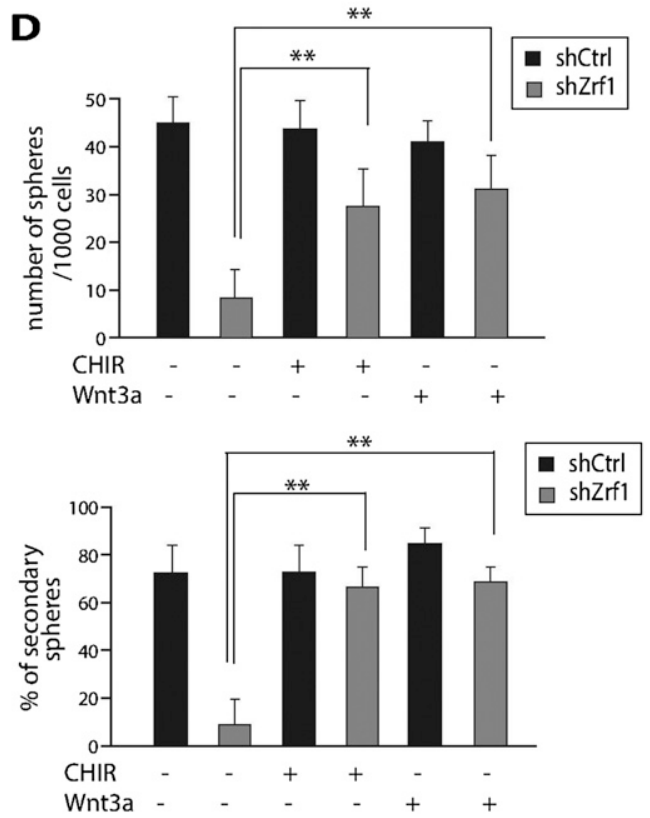

Figure 6. Zrf1-mediated control of Wnt signaling is essential for NPC self-renewal. (A, left) Western blot of active and total $\beta$-catenin, Pax6, and Zrf1 in shCtrl or shZrf1 NPCs treated or not with CHIR. (Right) Band intensity was quantified using the ImageJ software. Data represent the average of three independent experiments. $\left(^{\star}\right) P<0.05 ;\left(^{\star \star}\right) P<0.01 .(B)$ Pax6 mRNA expression levels in shCtrl and shZrf1 NPCs treated with CHIR or Wnt3a. $(C)$ Representative pictures of spheres derived from shCtrl and shZrf1 NPCs treated with CHIR or Wnt3a. Bar, $150 \mu \mathrm{m}$. (D, top panel) Number of shCtrl and shZrf1 NPCs treated with CHIR, Wnt3a, or DMSO (as a control) that gave rise to primary spheres over 1000 cells. (Bottom panel) The percentage of cells derived by disaggregation of primary spheres able to give rise to secondary spheres over the total number of cells. Data are representative of five different experiments. $\left(^{\star \star}\right) P<0.01$.

found that several markers of RGC identity, including Pax6 and Sox2, were down-regulated after Zrf1 depletion at both the transcriptional and protein levels (Fig. 7D; Supplemental Fig. S8D). Interestingly, Sox2 and Pax6 expression was also diminished in surrounding nonelectroporated cells (Supplemental Fig. S8D), suggesting that Zrf1 depletion also exerts a non-cell-autonomous effect. Furthermore, in line with our data from cultured NPCs, mRNA levels of several Wnt ligands were down-regulated in shZrf1 cells (Fig. 7D), suggesting that impairment of autocrine/paracrine Wnt signaling could be responsible for the non-cell-autonomous effect due to Zrf1 depletion. Re-expression of Zrf1 using a human Zrf1 cDNA construct restored proper levels of $\mathrm{Pax} 6$ as well as of the other RGC markers, including Wnt ligand genes (Fig. 7D; Supplemental Fig. S8D). Finally, we also analyzed the expression of $\beta 3$-tubulin, a marker of post-mitotic neurons, in the electroporated regions of the embryonic cortex. Surprisingly, immunostaining analysis indicated that $\beta 3$-tubulin-positive neurons aberrantly accumulated in the VZ in Zrf1-depleted cells and their surrounding cells (Supplemental Fig. S8E), suggesting that Zrf1 depletion induced 
Aloia et al.

A

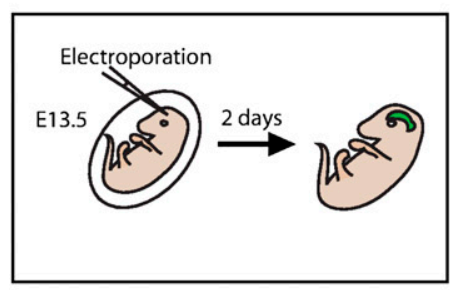

B

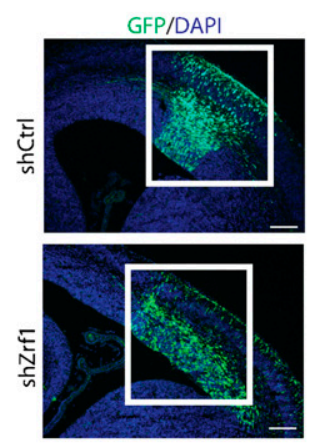

C

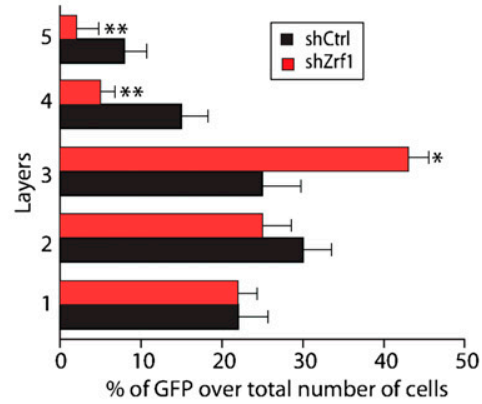

D
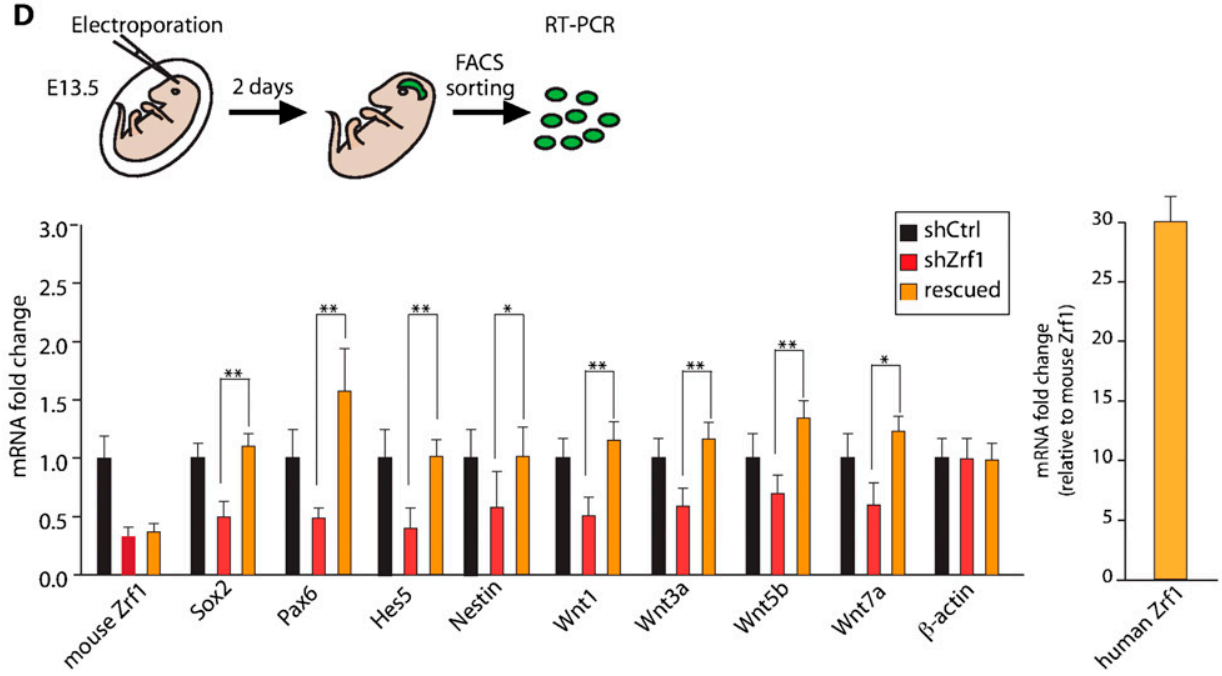

$\mathbf{E}$

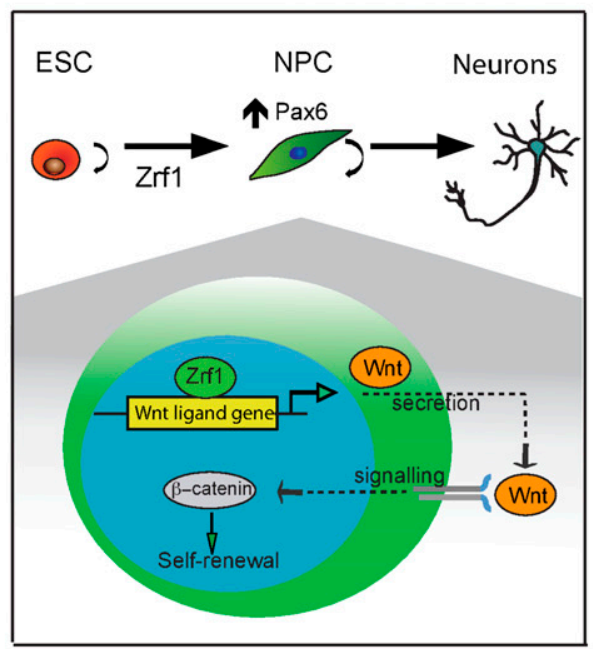

Figure 7. Zrf1 regulates the expression of Wnt ligands and self-renewal regulators in vivo. $(A)$ Scheme of the experimental procedure. (B) Coronal hemisections of forebrains $48 \mathrm{~h}$ after electroporation with plasmids containing shCtrl or shZrf1. Bars, $100 \mu \mathrm{m}$. $(C)$ Percentage of electroporated cells (GFP-positive) in five layers of the embryonic cortex $48 \mathrm{~h}$ after electroporation. $\left(^{\star}\right) P<0.05 ;\left({ }^{\star \star}\right) P<$ 0.01. (D) GFP-positive cells were isolated by FACS $48 \mathrm{~h}$ after electroporation with shCtrl, shZrf1, or shZrf1 + human Zrf1 cDNA in the VZ/subventricular zone (SVZ) of an E13.5 embryonic cortex. The relative mRNA expression of RGC markers and selected Wnt ligands is reported in the graph. Values are normalized to Gapdh. Data represent the average of three independent experiments (FACS-sorted cells from three different embryos were pooled together for each experiment). $\left(^{\star}\right) P<0.05 ;\left(^{\star \star}\right) P<0.01$. (E) Model: Zrf1 is essential to generate Pax6-positive NPCs from ESCs. In NPCs, Zrf1 directly regulates the expression of several Wnt ligand genes, which in turn initiate autocrine/paracrine canonical Wnt signaling, responsible for maintaining NPC self-renewal. 
premature differentiation in a significant fraction of RGCs. Taken together, these data highlight a role of Zrf1 as key regulator of NPC features in vivo.

\section{Discussion}

Our data show a specific role of the chromatin-binding factor Zrf1 in neural lineage specification and the selfrenewal capability of Pax6-positive NPCs (summarized in Fig. 7E). Our earlier work had shown that Zrf1 displaces the Polycomb proteins of the PRC1 complex from promoters upon differentiation of human teratocarcinoma lines (Richly et al. 2010). Here we show that Zrf1 is specifically involved in neural fate, as Zrf1 depletion did not affect mesodermal and endodermal specification from ESCs or the stem cell features of several nonneural stem cell lineages. Furthermore, ChIP-seq data revealed that a PRC1-dependent role of Zrf1 is required for neurogenesis at a genome-wide level. Our data thus expand the molecular characterization of epigenetic regulation of neural development mediated by Polycomb proteins (Mohn et al. 2008; Hirabayashi et al. 2009; Aloia et al. 2013). At the biochemical level, our previous data in human teratocarcinoma cells indicate that, in differentiation conditions, Zrf1 can displace PRC1 from chromatin by competing for the binding to monoubiquitinated H2A. However, we cannot exclude that Zrf1 recruitment to chromatin upon NPC specification actually promotes NPC generation, which eventually leads to the displacement of the PRC1 complex from its ESC targets. Importantly, our ChIP-seq data suggest that Zrf1 also exerts a Polycomb-independent role that should be explored more in detail in the future.

At a molecular level, we show here that Zrf1 sustains the expression of the key self-renewal regulator Pax6 and that re-expression of Pax6 restores the self-renewal capability of Zrf1-depleted NPCs. Although several reports have elucidated the downstream targets of Pax6 (Sansom et al. 2009; Ninkovic et al. 2013; Walcher et al. 2013), so far, little is known about the regulation of Pax6 expression. A possible mechanism is via the Wnt signaling pathway, which has also been reported to be crucial for NPC self-renewal (Chenn and Walsh 2002; Kalani et al. 2008; Wexler et al. 2009). We found that Zrf1 depletion induced a significant decrease in the amount of active $\beta$-catenin, the downstream effector of the canonical Wnt pathway. Treatment with the GSK3-kinase inhibitor CHIR partially restored Pax6 expression and the selfrenewal capacity of Zrf1-depleted NPCs, suggesting that Pax6 could be a downstream target of the Wnt signaling in NPCs. How does Zrf1 depletion induce a decrease in the levels of active $\beta$-catenin? We found that Zrf1 is essential for the establishment and maintenance of correct levels of selected Wnt ligands in NPCs. Although autocrine/paracrine Wnt signaling sustained by endogenously produced Wnt ligands has been previously reported to be crucial for NPC self-renewal (Chenn and Walsh 2002; Kalani et al. 2008; Wexler et al. 2009), molecular mechanisms regulating the expression of Wnt ligands in NPCs have not yet been studied in detail.
Our finding that Zrf1 mediated the regulation of the Wnt ligands is in apparent contrast to the neurogenic function of Zrf1, as previous reports indicate that the Wnt signaling impairs neural induction from ESCs (Aubert et al. 2002; Haegele et al. 2003; Cajanek et al. 2009; Blauwkamp et al. 2012). However, in physiological conditions, we found that the levels of active $\beta$-catenin expressed were lower during neural specification than in either ESCs or self-renewing NPCs, suggesting that induction of selected Wnt ligands could be counteracted by specific inhibitors (Aubert et al. 2002; Haegele et al. 2003; Cajanek et al. 2009).

We also observed that Zrf1 is required in vivo for selfrenewal of embryonic neural progenitors (e.g., RGCs). Importantly, only few differences were observed in vitro and in vivo, the most evident of which was the significant down-regulation of Sox 2 after Zrf1 depletion in vivo. This discrepancy could be due to the high concentration of EGF in the NPC culture medium, which strongly sustains Sox2 expression and might compensate for any effects of Zrf1 depletion in cultured NPCs (Hu et al. 2010). The decreased level of Sox 2 observed after introducing shZrf1 to the embryonic cortex could be responsible for the premature differentiation of RGCs into $\beta 3$-tubulin-positive neurons, as previously described (Estivill-Torrus et al. 2002; Tiberi et al. 2012). On the other hand, in cultured NPCs, we observed a significant decrease in $\beta 3$-tubulin staining in conditions that promoted neuronal differentiation. We therefore do not believe that $\beta 3$-tubulin up-regulation in shZrf1 self-renewing RGCs is in contrast with our data regarding the proneurogenic function of Zrf1 in cultured NPCs.

NPCs/neural stem cells have recently been used for tissue regeneration in model systems and are highly promising for developing treatments for neurodegenerative diseases (Temple 2001; Aboody et al. 2011). Moreover, the recent finding that mouse embryonic fibroblasts (MEFs) can be reprogrammed into induced NPCs/neural stem cells has opened new horizons in regenerative medicine (Han et al. 2012; Lujan et al. 2012; Ring et al. 2012; Thier et al. 2012). In this regard, our data suggest that, by developing strategies to recruit Zrf1 to chromatin and sustain its transcriptional effect, Zrf1 is a potential target for regenerative medicine. As Zrf1 has been shown previously to be involved in oncogene-induced senescence (Ribeiro et al. 2013) and misregulated in several human tumors (Shoji et al. 1995; Resto et al. 2000; Greiner et al. 2003), we suggest that Zrf1 could also play a role in brain tumor development. How Zrfl is recruited to chromatin and, once there, specifically regulates different processes (senescence, specification of NPCs, NPC maintenance, and differentiation) remains to be addressed. One possibility is that post-translational modifications of Zrf1 could confer the specificity to Zrf1 in different cell contexts, culture conditions, and developmental stages. Indeed, Zrf1 is phosphorylated upon RA in P19 cells (Smith et al. 2007). Another possibility is that the presence of specific interactors could specifically restrict Zrf1 function at different developmental stages. For instance, the protein inhibitor of DNA binding (Id1) 
has been shown previously to interact with Zrf1 (Richly et al. 2010).

Taken together, our data show that Zrf1 is a crucial player involved in NPC specification. Initially, Zrf1 acts through epigenetic processes to regulate the establishment of NPC fate. Once established, Zrf1 continues to act via the Wnt signaling pathway and the neural transcriptional network to maintain NPC identity.

\section{Materials and methods}

\section{Vectors}

The sequences of lentiviral shRNA \#1/2 (plKO, Sigma) are listed in Supplemental Table S2. shCtrls were from Sigma.

For rescue experiments in ESCs and NPCs, human Zrf1 cDNA was cloned in the pCBA-3xFlag vector (Aloia et al. 2010). For rescue in the in vivo electroporation experiments, Zrf1 was cloned in a pCAG-GFP vector (Sessa et al. 2008). For Pax6 overexpression, mouse Pax6 cDNA (NM_013627) was cloned in pCBA-3xFlag vector.

\section{ESC culture}

E14Tg2A and Sox1-GFP ESC lines (a kind gift from Dr. Smith) (Ying et al. 2003) were cultured as previously described (Aloia et al. 2010).

For alkaline phosphatase staining (Stemgent), 300 cells were plated per six-well plate and grown for $6 \mathrm{~d}$. ESCs stably expressing Zrf1 shRNA \#1/2/3 were generated by infection with lentiviral vectors and then selected with puromycin as previously described (Morey et al. 2012). To obtain Zrf1-Flag ESCs, cells were transfected with pCBA Zrf1-3 $\times$ Flag vector using Lipofectamine 2000 according to the manufacturer's instructions. Cells were then selected with neomycin for $7 \mathrm{~d}$.

\section{ESC lineage specification}

For neuroectodermal specification, ESCs were cultured in serum-free medium supplemented with N2 and B27 as previously described (Ying et al. 2003). RGC-like NPCs were obtained by culturing ESCs as cell aggregates with RA as previously described (Bibel et al. 2004, 2007). Endodermal and mesodermal lineages were created by treating ESCs for $5 \mathrm{~d}$ with activin A $(20$ $\mathrm{ng} / \mathrm{mL}$ ) as previously reported (Fei et al. 2010). Self-renewing NPCs were derived from ESCs as previously described (Colombo et al. 2006).

\section{NPC culture}

NPCs derived from ESCs were cultured in medium containing $20 \mathrm{ng} / \mathrm{mL}$ EGF and $10 \mathrm{ng} / \mathrm{mL}$ FGF2 as previously described (Colombo et al. 2006). Zrf1 and Pax6 re-expression was obtained by electropoating pCBA Zrf1 3xFlag and pCBA Pax6 3xFlag (Amaxa neural stem cell kit), and selected with neomycin for $6 \mathrm{~d}$. NPCs were treated with $3 \mu \mathrm{M}$ CHIR (Deltaclon) or $100 \mathrm{ng} / \mathrm{mL}$ Wnt3a (R\&D Systems) for $7 \mathrm{~d}$, replacing fresh medium daily, as indicated. Sphere formation was obtained by plating 10,000 cells in a six-well plate (without adherent) for cell culture. Secondary spheres were generated by disaggregating primary spheres and plating a single cell in a 96-well plate. For differentiation toward post-mitotic neurons, NPCs were cultured in N2 medium without EGF or FGF2 for $5 \mathrm{~d}$ or with N2 medium supplemented with B27 without EGF or FGF2 for $7 \mathrm{~d}$.

\section{MSC, HPC7, and C2C12 culture and differentiation}

$\mathrm{HPC} 7$ and $\mathrm{C} 2 \mathrm{C} 12$ were cultured and differentiated as previously published (Pinto do O et al. 1998; Burattini et al. 2004). MSCs (Life S1505-100) were grown and differentiated as previously described (Meirelles Lda and Nardi 2003).

\section{ChIP assay}

ChIP assays were performed as previously described (Morey et al. 2012) using anti-Zrfl and anti-RinglB antibodies (Richly et al. 2010). Primers used for ChIP-qPCR analysis are listed in Supplemental Table S3. Reads produced by ChIP-seq experiments of each of the samples were aligned with the mouse genome (version NCBIM37) using the Bowtie tool (version 0.12.7), allowing for two mismatches within the seed alignment. Sequence tags aligned to the genome were subsequently analyzed with MACS software (version 1.4.1) to detect genomic regions enriched for multiple overlapping DNA fragments (peaks) that we considered to be putative binding sites. MACS estimated the FDR by comparing the peaks obtained from the samples with those from the control samples using the same $P$ cutoff $\left(1 \times 10^{-5}\right)$. For this study, we considered all of the peaks $<10 \%$ FDR for Ring1B in ESCs, $1 \%$ FDR for Ring1B in NPCs, and 5\% FDR for Zrf1 in NPCs. We used the Ensembl annotation (version 63) to annotate the peaks and detect the closest TSS. ChIP-seq signals around TSSs were calculated using BEDTools over TSS overlapping peaks and normalizing the coverage for the total number of mapped reads. The Gene Expression Omnibus (GEO) accession number for this set of data is GSE53542.

\section{Western blots}

Proteins were extracted as previously described (Aloia et al. 2010). Antibodies used for Western blot were against Zrf1 (Richly et al. 2010), Pax6 (1:250; Millipore), Gapdh (1:2000; Santa Cruz Biotechnology), $\beta 3$-tubulin (1:300; Sigma), total $\beta$-catenin (1:2000; BD Biosciences), active $\beta$-catenin (1:1000; Millipore), Flag (1:500; Sigma), and Wnt1 (1:250; Sigma).

\section{RNA extraction and gene expression profile}

RNA was extracted with an RNA extraction kit (Qiagen). cDNA was generated from $1 \mathrm{mg}$ of RNA with the First Strand cDNA synthesis kit (Fermentas). Primers used in the RT-qPCR assays are listed in Supplemental Table S3. For gene expression profiles, RNA from four independent experiments was isolated and processed as previously described (Morey et al. 2012). A cutoff of \pm 2.0 -fold with $P \leq 0.01$ was used to determine differentially expressed genes. The GEO accession number for this set of data is GSE53542.

\section{FACS analysis}

Cell suspensions were analyzed for GFP expression or stained with antibodies against SSEA1 (FAB2155A, R\&D Systems), Mac-1 (clone M1/70, BD Biosciences), or Gr1 (clone RB6-8C5, BD Biosciences). Cells were analyzed with an LSR II FACS (BD Biosciences) using Diva version 6.1.2 (BD Biosciences) and FlowJo software version 10.0.6

\section{Cell immunofluorescence}

Cells were fixed in $4 \%$ paraformaldehyde and permeabilized with $0.2 \%$ Triton X-100 in $10 \%$ FBS (Invitrogen)/ $1 \%$ BSA in PBS for $15 \mathrm{~min}$ at room temperature. Samples were then incubated with primary antibodies overnight. The antibody used for cell 
immunofluorescence was against $\beta 3$-tubulin (1:300; Sigma) or eMHC (F1.652, Hybridoma Bank). Images were captured with an inverted microscope (DMI4000, Leica Microsystems).

\section{Teratoma formation}

SCID mice were injected subcutaneously with $5 \times 10^{5}$ ESCs infected with shCtrl or shZrf1 \#1 and \#2. Four weeks after the injection, tumors were surgically dissected from the mice, homogenized (for total RNA was extracted) or embedded in OCT (Tissue-Tek) and frozen at $-80^{\circ} \mathrm{C}$. Sections were stained with Nestin (1:300; Millipore), $\beta 3$-tubulin (1:200; Covance), GFAP (1:300; Dako), SMA (1:200; Sigma), or Foxa2 (1:100; Abcam) antibodies.

\section{In vivo electroporation}

Electroporation was used to deliver expression vectors or reporter constructs to the ventricular RGCs in utero as previously described (Saito and Nakatsuji 2001; Sessa et al. 2008, 2010). Briefly, uterine horns of E13.5 pregnant dams were exposed by midline laparotomy after anesthetization with $312 \mathrm{mg} / \mathrm{kg}$ Avertin. DNA plasmid ( $1 \mathrm{~mL}$, corresponding to $3 \mathrm{mg}$ ) mixed with $0.03 \%$ fast-green dye in PBS was injected into the telencephalic vesicle using a micropipette pulled through the uterine wall and amniotic sac. Platinum tweezer-style electrodes $(7 \mathrm{~mm})$ were placed outside the uterus over the telencephalon, and four pulses of $40 \mathrm{mV}$ for $50 \mathrm{msec}$ were applied at $950 \mathrm{msec}$ intervals using a BTX square wave electroporator. The uterus was then placed back into the abdomen, the cavity was filled with warm sterile PBS, and the abdominal muscle and skin incisions were closed with silk sutures.

\section{Immunohistochemistry of embryonic cortices}

Tissues were fixed with $4 \%$ paraformaldeheyde overnight at $4^{\circ} \mathrm{C}$, treated with $30 \%$ sucrose overnight at $4^{\circ} \mathrm{C}$, and then set to the optimal cutting temperature for cryosectioning. Immunochemistry analyses were performed as previously described (Colombo et al. 2004). Briefly, fixed cells or frozen sections were blocked in $10 \%$ normal goat serum (Sigma) and $0.1 \%$ Triton X-100 (Sigma) for $1 \mathrm{~h}$ at room temperature. Cells or the sections were then left overnight at $4^{\circ} \mathrm{C}$ with the primary antibody in blocking solution. Secondary antibodies were applied to cells or sections for $2 \mathrm{~h}$ at room temperature. The following primary antibodies were used: chicken anti-GFP (1:500; Molecular Probes), rabbit anti- $\beta 3$ tubulin (1:500; Covance), mouse anti-Sox2 (1:200; R\&D Systems), and rabbit Pax6 (1:200; R\&D Systems). Secondary antibodies (Molecular Probes) were conjugated with Alexa Fluor 488, Alexa Fluor 594, or Alexa Fluor 647 (1:500). DAPI (40,60diamidino-2-phenylindole) was used for nuclear counterstaining.

\section{Acknowledgments}

We are grateful to Dr. Veronica A. Raker for critical reading of the manuscript, and Dr. Austin Smith for Sox1-GFP cells. We also thank Dr. Pedro Vizán, Dr. Malte Beringer, Dr. Pia Cosma, Dr. Luigi Ombrato, and Dr. Serena Mirra for helpful discussions. We are indebted to the CRG animal facility, CRG FACS facility, and CRG microarray and genomic facility. This work was supported by grants from the Spanish "Ministerio de Educación y Ciencia" (BFU2010-18692), the Agència de Gestió d'Ajuts Universitaris i de Recerca (AGAUR), the American Institute for Cancer Research (AICR) (10-0177), and the European Commission's 7th Framework Program 4DCellFate (grant no. 277899) to L.D.C., and the AdERC (grant 340527 ) to V.B. B.D.S has a la Caixa International PhD fellowship, and A.S. has a Formación de Personal Investigador (FPI) PhD fellowship.

\section{References}

Aboody K, Capela A, Niazi N, Stern JH, Temple S. 2011. Translating stem cell studies to the clinic for CNS repair: Current state of the art and the need for a Rosetta stone. Neuron 70: 597-613.

Aloia L, Parisi S, Fusco L, Pastore L, Russo T. 2010. Differentiation of embryonic stem cells 1 (Dies1) is a component of bone morphogenetic protein 4 (BMP4) signaling pathway required for proper differentiation of mouse embryonic stem cells. J Biol Chem 285: 7776-7783.

Aloia L, Di Stefano B, Di Croce L. 2013. Polycomb complexes in stem cells and embryonic development. Development 140: 2525-2534.

Alvarez-Medina R, Le Dreau G, Ros M, Marti E. 2009. Hedgehog activation is required upstream of Wnt signalling to control neural progenitor proliferation. Development 136: 33013309.

Aubert J, Dunstan H, Chambers I, Smith A. 2002. Functional gene screening in embryonic stem cells implicates Wnt antagonism in neural differentiation. Nat Biotechnol 20: 1240-1245.

Aubert J, Stavridis MP, Tweedie S, O'Reilly M, Vierlinger K, Li M, Ghazal P, Pratt T, Mason JO, Roy D, et al. 2003. Screening for mammalian neural genes via fluorescence-activated cell sorter purification of neural precursors from Soxl-gfp knockin mice. Proc Natl Acad Sci 100: 11836-11841.

Bani-Yaghoub M, Tremblay RG, Lei JX, Zhang D, Zurakowski B, Sandhu JK, Smith B, Ribecco-Lutkiewicz M, Kennedy J, Walker PR, et al. 2006. Role of Sox2 in the development of the mouse neocortex. Dev Biol 295: 52-66.

Bibel M, Richter J, Schrenk K, Tucker KL, Staiger V, Korte M, Goetz M, Barde YA. 2004. Differentiation of mouse embryonic stem cells into a defined neuronal lineage. Nat Neurosci 7: 1003-1009.

Bibel M, Richter J, Lacroix E, Barde YA. 2007. Generation of a defined and uniform population of CNS progenitors and neurons from mouse embryonic stem cells. Nat Protoc 2: 1034-1043.

Blauwkamp TA, Nigam S, Ardehali R, Weissman IL, Nusse R. 2012. Endogenous Wnt signalling in human embryonic stem cells generates an equilibrium of distinct lineage-specified progenitors. Nat Commun 3: 1070.

Bluske KK, Vue TY, Kawakami Y, Taketo MM, Yoshikawa K, Johnson JE, Nakagawa Y. 2012. $\beta$-Catenin signaling specifies progenitor cell identity in parallel with Shh signaling in the developing mammalian thalamus. Development 139: 26922702.

Boiani M, Scholer HR. 2005. Regulatory networks in embryoderived pluripotent stem cells. Nat Rev Mol Cell Biol 6: 872 884.

Bowman AN, van Amerongen R, Palmer TD, Nusse R. 2013. Lineage tracing with Axin2 reveals distinct developmental and adult populations of Wnt/ $\beta$-catenin-responsive neural stem cells. Proc Natl Acad Sci 110: 7324-7329.

Burattini S, Ferri P, Battistelli M, Curci R, Luchetti F, Falcieri E. 2004. C2C12 murine myoblasts as a model of skeletal muscle development: Morpho-functional characterization. Eur I Histochem 48: 223-233.

Cajanek L, Ribeiro D, Liste I, Parish CL, Bryja V, Arenas E. 2009. Wnt/ $\beta$-catenin signaling blockade promotes neuronal induction and dopaminergic differentiation in embryonic stem cells. Stem Cells 27: 2917-2927. 
Campbell K, Gotz M. 2002. Radial glia: Multi-purpose cells for vertebrate brain development. Trends Neurosci 25: 235238.

Chenn A, Walsh CA. 2002. Regulation of cerebral cortical size by control of cell cycle exit in neural precursors. Science 297: 365-369.

Colombo E, Galli R, Cossu G, Gecz J, Broccoli V. 2004. Mouse orthologue of ARX, a gene mutated in several X-linked forms of mental retardation and epilepsy, is a marker of adult neural stem cells and forebrain GABAergic neurons. Dev Dyn 231: 631-639.

Colombo E, Giannelli SG, Galli R, Tagliafico E, Foroni C, Tenedini E, Ferrari S, Corte G, Vescovi A, Cossu G, et al. 2006. Embryonic stem-derived versus somatic neural stem cells: A comparative analysis of their developmental potential and molecular phenotype. Stem Cells 24: 825-834.

Conti L, Pollard SM, Gorba T, Reitano E, Toselli M, Biella G, Sun Y, Sanzone S, Ying QL, Cattaneo E, et al. 2005. Nicheindependent symmetrical self-renewal of a mammalian tissue stem cell. PLoS Biol 3: e283.

Estivill-Torrus G, Pearson H, van Heyningen V, Price DJ, Rashbass P. 2002. Pax6 is required to regulate the cell cycle and the rate of progression from symmetrical to asymmetrical division in mammalian cortical progenitors. Development 129: 455-466.

Fei T, Zhu S, Xia K, Zhang J, Li Z, Han JD, Chen YG. 2010. Smad2 mediates Activin/Nodal signaling in mesendoderm differentiation of mouse embryonic stem cells. Cell Res 20: $1306-1318$.

Fuentealba LC, Obernier K, Alvarez-Buylla A. 2012. Adult neural stem cells bridge their niche. Cell Stem Cell 10: 698-708.

Gomez-Lopez S, Wiskow O, Favaro R, Nicolis SK, Price DI, Pollard SM, Smith A. 2011. Sox2 and Pax6 maintain the proliferative and developmental potential of gliogenic neural stem cells in vitro. Glia 59: 1588-1599.

Gotz M, Huttner WB. 2005. The cell biology of neurogenesis. Nat Rev Mol Cell Biol 6: 777-788.

Gotz M, Stoykova A, Gruss P. 1998. Pax6 controls radial glia differentiation in the cerebral cortex. Neuron 21: 1031-1044.

Greiner J, Ringhoffer M, Taniguchi M, Hauser T, Schmitt A, Dohner H, Schmitt M. 2003. Characterization of several leukemia-associated antigens inducing humoral immune responses in acute and chronic myeloid leukemia. Int $J$ Cancer 106: 224-231.

Haegele L, Ingold B, Naumann H, Tabatabai G, Ledermann B, Brandner S. 2003. Wnt signalling inhibits neural differentiation of embryonic stem cells by controlling bone morphogenetic protein expression. Mol Cell Neurosci 24: 696-708.

Han YG, Spassky N, Romaguera-Ros M, Garcia-Verdugo JM, Aguilar A, Schneider-Maunoury S, Alvarez-Buylla A. 2008. Hedgehog signaling and primary cilia are required for the formation of adult neural stem cells. Nat Neurosci 11: 277-284.

Han DW, Tapia N, Hermann A, Hemmer K, Hoing S, ArauzoBravo MJ, Zaehres H, Wu G, Frank S, Moritz S, et al. 2012. Direct reprogramming of fibroblasts into neural stem cells by defined factors. Cell Stem Cell 10: 465-472.

Hatzold J, Conradt B. 2008. Control of apoptosis by asymmetric cell division. PLOS Biol 6: e84.

Heins N, Malatesta P, Cecconi F, Nakafuku M, Tucker KL, Hack MA, Chapouton P, Barde YA, Gotz M. 2002. Glial cells generate neurons: The role of the transcription factor Pax6. Nat Neurosci 5: 308-315.

Hirabayashi $\mathrm{Y}$, Itoh $\mathrm{Y}$, Tabata $\mathrm{H}$, Nakajima $\mathrm{K}$, Akiyama $\mathrm{T}$, Masuyama N, Gotoh Y. 2004. The Wnt/ß-catenin pathway directs neuronal differentiation of cortical neural precursor cells. Development 131: 2791-2801.

Hirabayashi Y, Suzki N, Tsuboi M, Endo TA, Toyoda T, Shinga I, Koseki H, Vidal M, Gotoh Y. 2009. Polycomb limits the neurogenic competence of neural precursor cells to promote astrogenic fate transition. Neuron 63: 600-613.

Hu Q, Zhang L, Wen J, Wang S, Li M, Feng R, Yang X, Li L. 2010. The EGF receptor-sox2-EGF receptor feedback loop positively regulates the self-renewal of neural precursor cells. Stem Cells 28: 279-286.

Kalani MY, Cheshier SH, Cord BJ, Bababeygy SR, Vogel H, Weissman IL, Palmer TD, Nusse R. 2008. Wnt-mediated selfrenewal of neural stem/progenitor cells. Proc Natl Acad Sci 105: 16970-16975.

Kriegstein A, Alvarez-Buylla A. 2009. The glial nature of embryonic and adult neural stem cells. Annu Rev Neurosci 32: 149-184.

Lee JE. 1997. Basic helix-loop-helix genes in neural development. Curr Opin Neurobiol 7: 13-20.

Lie DC, Colamarino SA, Song HJ, Desire L, Mira H, Consiglio A, Lein ES, Jessberger S, Lansford H, Dearie AR, et al. 2005. Wnt signalling regulates adult hippocampal neurogenesis. Nature 437: 1370-1375.

Lujan E, Chanda S, Ahlenius H, Sudhof TC, Wernig M. 2012. Direct conversion of mouse fibroblasts to self-renewing, tripotent neural precursor cells. Proc Natl Acad Sci 109: 2527-2532.

Lyashenko N, Winter M, Migliorini D, Biechele T, Moon RT, Hartmann C. 2011. Differential requirement for the dual functions of $\beta$-catenin in embryonic stem cell self-renewal and germ layer formation. Nat Cell Biol 13: 753-761.

MacDonald BT, Tamai K, He X. 2009. Wnt/ $\beta$-catenin signaling: Components, mechanisms, and diseases. Dev Cell 17: 9-26.

Malatesta P, Hack MA, Hartfuss E, Kettenmann H, Klinkert W, Kirchhoff F, Gotz M. 2003. Neuronal or glial progeny: Regional differences in radial glia fate. Neuron 37: 751-764.

Malatesta P, Appolloni I, Calzolari F. 2008. Radial glia and neural stem cells. Cell Tissue Res 331: 165-178.

Meirelles Lda S, Nardi NB. 2003. Murine marrow-derived mesenchymal stem cell: Isolation, in vitro expansion, and characterization. Br J Haematol 123: 702-711.

Merkle FT, Mirzadeh Z, Alvarez-Buylla A. 2007. Mosaic organization of neural stem cells in the adult brain. Science 317: 381-384.

Miller FD, Gauthier-Fisher A. 2009. Home at last: Neural stem cell niches defined. Cell Stem Cell 4: 507-510.

Mizutani K, Saito T. 2005. Progenitors resume generating neurons after temporary inhibition of neurogenesis by Notch activation in the mammalian cerebral cortex. Development 132: $1295-1304$.

Mohn F, Weber M, Rebhan M, Roloff TC, Richter J, Stadler MB, Bibel M, Schübeler D. 2008. Lineage-specific polycomb targets and de novo DNA methylation define restriction and potential of neuronal progenitors. Mol Cell 30: 755766.

Morey L, Pascual G, Cozzuto L, Roma G, Wutz A, Benitah SA, Di Croce L. 2012. Nonoverlapping functions of the Polycomb group Cbx family of proteins in embryonic stem cells. Cell Stem Cell 10: 47-62.

Nichols J, Smith A. 2012. Pluripotency in the embryo and in culture. Cold Spring Harb Perspect Biol 4: a008128.

Ninkovic J, Steiner-Mezzadri A, Jawerka M, Akinci U, Masserdotti G, Petricca S, Fischer J, von Holst A, Beckers J, Lie CD, et al. 2013. The BAF complex interacts with Pax6 in adult neural progenitors to establish a neurogenic cross-regulatory transcriptional network. Cell Stem Cell 13: 403-418.

Niwa H. 2007. How is pluripotency determined and maintained? Development 134: 635-646. 
Nusse R. 2008. Wnt signaling and stem cell control. Cell Res 18: 523-527.

Otero JJ, Fu W, Kan L, Cuadra AE, Kessler JA. 2004. $\beta$-Catenin signaling is required for neural differentiation of embryonic stem cells. Development 131: 3545-3557.

Pinto do O P, Kolterud A, Carlsson L. 1998. Expression of the LIM-homeobox gene LH2 generates immortalized steel factor-dependent multipotent hematopoietic precursors. EMBO J 17: 5744-5756.

Resto VA, Caballero OL, Buta MR, Westra WH, Wu L, Westendorf JM, Jen J, Hieter P, Sidransky D. 2000. A putative oncogenic role for MPP11 in head and neck squamous cell cancer. Cancer Res 60: 5529-5535.

Ribeiro JD, Morey L, Mas A, Gutierrez A, Luis NM, Mejetta S, Richly H, Benitah SA, Keyes WM, Di Croce L. 2013. ZRF1 controls oncogene-induced senescence through the INK4ARF locus. Oncogene 32: 2161-2168.

Richly H, Rocha-Viegas L, Ribeiro JD, Demajo S, Gundem G, Lopez-Bigas N, Nakagawa T, Rospert S, Ito T, Di Croce L. 2010. Transcriptional activation of polycomb-repressed genes by ZRF1. Nature 468: 1124-1128.

Ring KL, Tong LM, Balestra ME, Javier R, Andrews-Zwilling Y, Li G, Walker D, Zhang WR, Kreitzer AC, Huang Y. 2012. Direct reprogramming of mouse and human fibroblasts into multipotent neural stem cells with a single factor. Cell Stem Cell 11: 100-109.

Saito T, Nakatsuji N. 2001. Efficient gene transfer into the embryonic mouse brain using in vivo electroporation. Dev Biol 240: 237-246.

Sansom SN, Griffiths DS, Faedo A, Kleinjan DJ, Ruan Y, Smith J, van Heyningen V, Rubenstein JL, Livesey FJ. 2009. The level of the transcription factor Pax6 is essential for controlling the balance between neural stem cell self-renewal and neurogenesis. PLoS Genet 5: e1000511.

Schuettengruber B, Cavalli G. 2009. Recruitment of polycomb group complexes and their role in the dynamic regulation of cell fate choice. Development 136: 3531-3542.

Sessa A, Mao CA, Hadjantonakis AK, Klein WH, Broccoli V. 2008. Tbr2 directs conversion of radial glia into basal precursors and guides neuronal amplification by indirect neurogenesis in the developing neocortex. Neuron 60: 56-69.

Sessa A, Mao CA, Colasante G, Nini A, Klein WH, Broccoli V. 2010. Tbr2-positive intermediate (basal) neuronal progenitors safeguard cerebral cortex expansion by controlling amplification of pallial glutamatergic neurons and attraction of subpallial GABAergic interneurons. Genes Dev 24: 18161826.

Shoji W, Inoue T, Yamamoto T, Obinata M. 1995. MIDA1, a protein associated with Id, regulates cell growth. I Biol Chem 270: 24818-24825.

Smith JC, Duchesne MA, Tozzi P, Ethier M, Figeys D. 2007. A differential phosphoproteomic analysis of retinoic acidtreated P19 cells. J Proteome Res 6: 3174-3186.

Stoykova A, Fritsch R, Walther C, Gruss P. 1996. Forebrain patterning defects in Small eye mutant mice. Development 122: 3453-3465.

Suter DM, Tirefort D, Julien S, Krause KH. 2009. A Sox1 to Pax6 switch drives neuroectoderm to radial glia progression during differentiation of mouse embryonic stem cells. Stem Cells 27: 49-58.

Temple S. 2001. The development of neural stem cells. Nature 414: 112-117.

Thier M, Worsdorfer P, Lakes YB, Gorris R, Herms S, Opitz T, Seiferling D, Quandel T, Hoffmann P, Nothen MM, et al. 2012. Direct conversion of fibroblasts into stably expandable neural stem cells. Cell Stem Cell 10: 473-479.
Tiberi L, van den Ameele J, Dimidschstein J, Piccirilli J, Gall D, Herpoel A, Bilheu A, Bonnefont J, Iacovino M, Kyba M, et al. 2012. BCL6 controls neurogenesis through Sirt1-dependent epigenetic repression of selective Notch targets. Nat Neurosci 15: 1627-1635.

Tuoc TC, Radyushkin K, Tonchev AB, Pinon MC, Ashery-Padan R, Molnar Z, Davidoff MS, Stoykova A. 2009. Selective cortical layering abnormalities and behavioral deficits in cortex-specific Pax6 knock-out mice. I Neurosci 29: 83358349.

Walcher T, Xie Q, Sun J, Irmler M, Beckers J, Ozturk T, Niessing D, Stoykova A, Cvekl A, Ninkovic J, et al. 2013. Functional dissection of the paired domain of Pax6 reveals molecular mechanisms of coordinating neurogenesis and proliferation. Development 140: 1123-1136.

Wexler EM, Paucer A, Kornblum HI, Palmer TD, Geschwind DH. 2009. Endogenous Wnt signaling maintains neural progenitor cell potency. Stem Cells 27: 1130-1141.

Ying QL, Stavridis M, Griffiths D, Li M, Smith A. 2003. Conversion of embryonic stem cells into neuroectodermal precursors in adherent monoculture. Nat Biotechnol 21: 183-186.

Ying QL, Wray J, Nichols J, Batlle-Morera L, Doble B, Woodgett I, Cohen P, Smith A. 2008. The ground state of embryonic stem cell self-renewal. Nature 453: 519-523.

Zhao C, Deng W, Gage FH. 2008. Mechanisms and functional implications of adult neurogenesis. Cell 132: 645-660. 


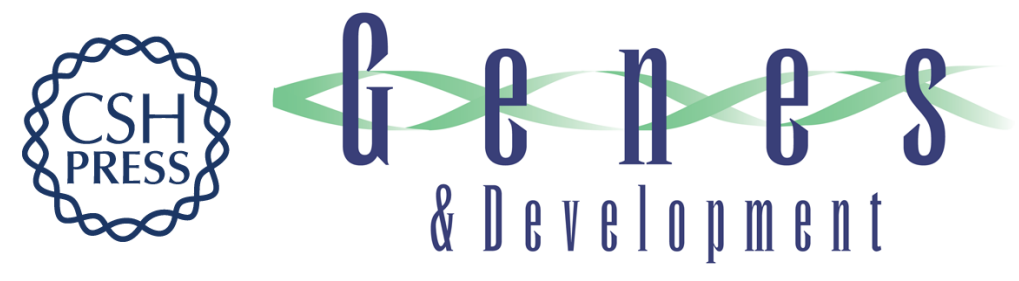

\title{
Zrf1 is required to establish and maintain neural progenitor identity
}

\author{
Luigi Aloia, Bruno Di Stefano, Alessandro Sessa, et al.
}

Genes Dev. 2014, 28:

Access the most recent version at doi:10.1101/gad.228510.113

\section{Supplemental http://genesdev.cshlp.org/content/suppl/2014/01/21/28.2.182.DC1 \\ Material}

References This article cites 78 articles, 23 of which can be accessed free at: http://genesdev.cshlp.org/content/28/2/182.full.html\#ref-list-1

Creative This article is distributed exclusively by Cold Spring Harbor Laboratory Press for the first Commons License six months after the full-issue publication date (see http://genesdev.cshlp.org/site/misc/terms.xhtml). After six months, it is available under a Creative Commons License (Attribution-NonCommercial 3.0 Unported), as described at http://creativecommons.org/licenses/by-nc/3.0/.

Email Alerting Receive free email alerts when new articles cite this article - sign up in the box at the top Service right corner of the article or click here.

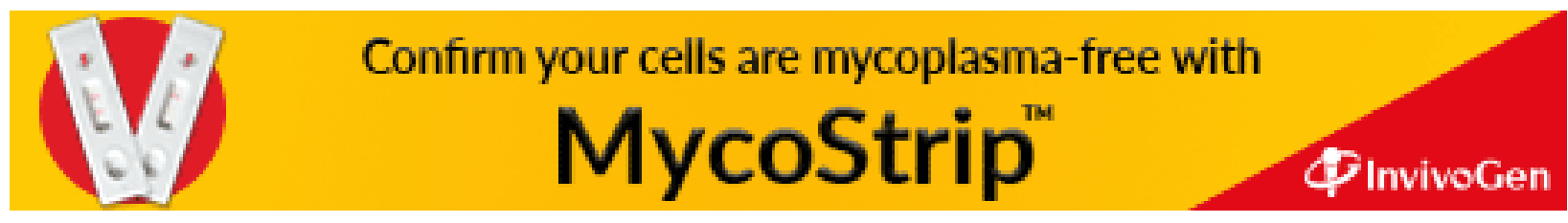

